\title{
Anterior thalamic nuclei: A critical substrate for non-spatial paired-associate memory
}

Jennifer J Hamilton ${ }^{1,2,3}$, John C Dalrymple-Alford ${ }^{1,2.3}$

1. School of Psychology, Speech and Hearing, University of Canterbury, 20 Kirkwood

Avenue, Christchurch 8041, New Zealand

2. New Zealand Brain Research Institute, 66 Stewart Street, Christchurch 8011, New Zealand

3. Brain Research New Zealand - Rangahau Roro Aotearoa

Correspondence: John C Dalrymple-Alford or Jennifer J Hamilton. University of Canterbury, School of Psychology, Speech and Hearing, 20 Kirkwood Avenue, Christchurch 8041, New Zealand. Tel.: +64 3364 2998; fax: +64 03364 2181. E-mail addresses:

jc.dalrymple.alford@gmail.com, john.dalrymple-alford@ canterbury.ac.nz,or, jjhamilton91@gmail.com, jjh75@uclive.ac.nz

Running title:

\section{ATN and non-spatial paired-associate memory}

Total 45 pages with 8 figures

Keywords: thalamic amnesia, biconditional discrimination, extended memory system, rat, immediate early genes. 


\section{Abstract}

The anterior thalamic nuclei (ATN), a central node in a complex memory system, process spatial and temporal memory. Here, we show that ATN lesions do not affect acquisition of a simple odour discrimination or a simple object discrimination in a runway apparatus. The same procedures were used to test learning of an arbitrary association between non-spatial object-odour pairings $(\mathrm{A}+\mathrm{X}$ or $\mathrm{B}+\mathrm{Y}$ were rewarded; but not $\mathrm{A}+\mathrm{Y}$ or $\mathrm{B}+\mathrm{X})$. If $\mathrm{ATN}$ lesions recapitulate hippocampal function, specifically CA1 function, then they should disrupt acquisition only when an explicit delay (i.e., a 10-second trace) is inserted between the odour and object. Acquisition was completely abolished by ATN lesions, irrespective of the presence of the temporal trace, and despite extensive training (50x12-trial sessions). Faster acquisition with the 10-second trace was found in the sham-lesion rats. During recall, 5 days after criterion, sham rats but not ATN-lesion rats showed elevated Zif268 expression in hippocampal CA1 for the trace compared to no-trace condition; both sham and lesion rats tested in the trace condition showed increased IEG expression in the superficial layers of the prefrontal cortex and retrosplenial cortex. ATN lesions markedly reduced Zif268 expression in the prefrontal cortex and retrosplenial cortex. This is the first evidence that ATN lesions impair non-spatial paired-associate tasks. The findings suggest that the ATN influence memory beyond time and space, and constitute a critical neural structure for learning arbitrary associations even in the task version that is not disrupted by hippocampal lesions. 


\section{Introduction}

The anterior thalamic nuclei (ATN) have complex reciprocal connections with many brain structures in a 'hippocampal-diencephalic-cingulate' network (Bubb, Kinnavane, \& Aggleton, 2017). The pattern of connections suggests that this small brain region is a critical subcortical node that supports episodic memory. This perspective aligns with evidence that loss of ATN neurons in people with the Korsakoff's syndrome, which is characterised by persistent and severe amnesia, discriminates these patients from alcoholic individuals who retain relatively good memory (Harding, Halliday, Caine, \& Kril, 2000; Kopelman, 2015; Maillard et al., 2021). Intra-thalamic recordings in epilepsy patients also reinforce the association between ATN function and memory (Sweeney-Reed et al., 2021). Additional clinical support comes from disruption to the ATN caused by mammillothalamic tract (MTT) lesions, which is the brain injury that most consistently corresponds with severe amnesia after thalamic infarcts (Carlesimo, Lombardi, \& Caltagirone, 2011).

This association between the ATN and memory is supported by a large body of experimental lesion studies. However, these animal studies have almost invariably focused on tests of memory for distal spatial cues (Aggleton \& Nelson, 2015; Dalrymple-Alford et al., 2015; Nelson, 2021; Perry, Mercer, Barnett, Lee, \& Dalrymple-Alford, 2018). Evidence for non-spatial memory impairment after experimental ATN lesions has been found after examining non-spatial judgements involving the temporal order of individual items (Wolff, Gibb, \& Dalrymple-Alford, 2006). These temporal memory deficits may, however, be restricted to more difficult discriminations among a list of multiple items within a single episode rather than unique pairs of items or when judgements are made across two separate sequences of multiple items (Aggleton, Amin, Jenkins, Pearce, \& Robinson, 2011; Dumont \& Aggleton, 2013; Mitchell \& Dalrymple-Alford, 2005; Wolff et al., 2006). 
Existing studies provide limited insight on the influence of the ATN for memory beyond temporal order or the spatial environment (Nelson, 2021). The key premise of episodic memory in the broadest sense is the binding of arbitrary stimuli as a multimodal representation that guides recollection. This arbitrary association is especially relevant when the memory event includes two or more explicit elements or attributes, such as place, context, time, response type, object, odour and taste (Crystal \& Smith, 2014; Eichenbaum \& Fortin, 2009; Hunsaker \& Kesner, 2018). One class of arbitrary association memory is the ability to discriminate a combination of cues relative to their component elements (McDonald et al., 1997). Neither ATN lesions nor hippocampal lesions affect these configural learning tasks when they rely on non-spatial cues (Chudasama, Bussey, \& Muir, 2001; McDonald et al., 1997; Moran \& Dalrymple-Alford, 2003; Ridley, Maclean, Young, \& Baker, 2002). Conditional learning tasks provide a second class of arbitrary associations. This time, one correct response is signalled by one stimulus and a second correct response is signalled by an alternate stimulus. Unlike hippocampal lesions, however, ATN lesions did not delay acquisition when either egocentric responses or non-egocentric spatial choices were signalled by a single visual cue (Sziklas \& Petrides, 2004, 2007).

Paired-associate and related biconditional discrimination learning tasks offer a more direct test of memory for arbitrary associations in animals. These tasks use stimulus pairings that have explicitly overlapping attributes, in which $\mathrm{A}+\mathrm{X}$ or $\mathrm{B}+\mathrm{Y}$ are rewarded but not $\mathrm{A}+\mathrm{Y}$ or $\mathrm{B}+\mathrm{X}$. That is, the unique combination of attributes defines the episode, not whether a combination of cues is contrasted with their elements or when the conditional relationship is determined by a single salient cue. Like hippocampal lesions (Gilbert \& Kesner, 2002, 2003; Hunsaker, Thorup, Welch, \& Kesner, 2006; Jo \& Lee, 2010; Lee \& Solivan, 2008; Sziklas \& 
Petrides, 2002), ATN lesions produce severe acquisition impairments for odour-place or object-place associations using these procedures (Dumont, Amin, \& Aggleton, 2014; Gibb, Wolff, \& Dalrymple-Alford, 2006; Sziklas \& Petrides, 1999). It is unlikely that these impairments are unduly influenced by the place attribute of the association memory because ATN lesions have little effect on simple spatial discriminations using a similar protocol in the same apparatus (Dumont et al., 2014; Gibb et al., 2006). Nonetheless, ATN lesions did not impair biconditional discriminations when local contextual cues were used that were either visual, thermal or floor texture (Dumont et al., 2014).

Together, this evidence suggests that that the inclusion of spatial or temporal attributes are necessary in order to reveal memory impairments after ATN lesions. Contrary to this expectation, we found evidence of impaired acquisition of a non-spatial object-odour association task after ATN lesions in preliminary work when no explicit spatial or temporal component was included (Bell, 2007). That task was modelled on Kesner's work (Gilbert \& Kesner, 2003) in which the paired cues were presented on a large cheeseboard apparatus. This choice may have introduced distracting elements, such as distal spatial cues, because even our intact rats took 8 weeks of training before acquisition began to emerge. The current study extended this work on object-odour memory by using a larger group of rats, a different apparatus and the option to include an explicit temporal delay in the paired-associate task. We, therefore, used a high-walled red-Perspex runway, as this limits distraction from irrelevant spatial cues, and examined the inclusion or not of an explicit 10 second "trace" (i.e. temporal component) between the sequentially presented odour and object stimuli. Hippocampal lesions prevent the acquisition of object-odour associative memory only when the task includes the 10 second trace between the two non-spatial stimuli, which was identified as dependent on the loss of CA1 neurons (Gilbert \& Kesner, 2002; Kesner, 
Hunsaker, \& Gilbert, 2005). There are no direct ATN connections with hippocampal pyramidal neurons (Bubb et al., 2017), but ATN lesions nonetheless reduce the number of CA1 dendritic spines (Harland, Collings, McNaughton, Abraham, \& Dalrymple-Alford, 2014) and often reduce hippocampal immediate early gene (IEG) activation (Dupire et al., 2013; Jenkins, Dias, Amin, \& Aggleton, 2002; Jenkins, Dias, Amin, Brown, \& Aggleton, 2002; Loukavenko, Wolff, Poirier, \& Dalrymple-Alford, 2015; Perry et al., 2018). We anticipated that ATN lesions would slow the acquisition of the standard odour-object pairedassociate learning task when trained in the runway, but abolish learning in the trace version of this task because of the additional temporal component. We complemented this work by examining Zif268 IEG expression in memory-related brain structures after a recall session conducted 5 days after the end of training.

Materials and Methods

\section{Animals and housing conditions}

Male Long-Evans rats (12 months old; average 630g) were housed in groups of three of four in Makrolon cages (48 x $28 \times 22 \mathrm{~cm}$ ) on a reversed 12-hour light-dark cycle (lights on at 8pm). Behavioural testing occurred in the dark phase of the light cycle, five sessions per week; observations confirmed that rats were relatively more active during the dark phase. Rats were maintained at $85 \%$ of free feeding body weight with water ad-libitum; food was ad-libitum prior to and after surgery for post-operative recovery. All procedures complied with approved guidelines from the University of Canterbury Animal Ethics Committee.

\section{Surgery}

Rats were anaesthetised with isoflurane (induction $4 \%$; maintenance $~ 2.5 \%$ ) and placed in a stereotaxic apparatus with atraumatic ear bars (Kopf, Tujunga, CA). The incisor bar was set 
to $-7.5 \mathrm{~mm}$ below interaural line to minimise fornix damage. In each hemisphere, two infusions were directed at the anteroventral nucleus (AV; upper and lower) and one infusion was directed at the anteromedial nucleus (AM). Each surgery used one of five anteriorposterior (AP) coordinates relative to an individual rat's Bregma-Lambda distance (all values in mm). For AV lesions, the AP coordinates were: -2.20 for B-L 6.9-7.2; -2.25 for B-L 7.3 to $7.6 ;-2.30$ for $\mathrm{B}-\mathrm{L} \geq 7.7$. The AV infusions were made at -5.68 and -5.73 below dura and \pm 1.52 lateral to the midline. The AM infusion was made $0.1 \mathrm{~mm}$ more anterior than the AV coordinate, at -5.76 below dura and \pm 1.16 lateral; the first $\mathrm{AM}$ lesion was made after completing all AV infusions and began contralateral to the last AV lesion site. $0.15 \mathrm{M} \mathrm{N}$ methyl-d-aspartate (NMDA; Sigma, Castle Hill, NSW) in 0.1M phosphate buffer (pH 7.20) was infused into each site at $0.04 \mathrm{ul}$ per minute via a $2.5 \mu \mathrm{L}$ Hamilton syringe (Reno, NV, USA) using a micro infusion pump (Stoelting, Wooddale, IL). The AV sites per hemisphere received 0.12 and $0.10 \mu \mathrm{L}$ for the dorsal and ventral sites, respectively; $0.06 \mu \mathrm{L}$ was used for each AM site. The syringe needle remained in situ for 3 minutes per site, post-infusion. Sham lesion surgeries used the same procedure but the needle was lowered to $1 \mathrm{~mm}$ above the upper $\mathrm{AV}$ and the $\mathrm{AM}$ lesion sites with no infusion given.

\section{Behavioural Tasks}

The 8-arm radial arm maze (RAM) was used to confirm the spatial memory impairment in ATN-lesioned rats following surgery. A runway apparatus (Figure 1) was used to train the rats on all non-spatial tasks; i.e. simple odour and simple object discrimination, and odourobject and odour-trace-object paired-associate tasks. Prior to surgery, food-restricted rats were habituated to the 8 -arm RAM, described below, to retrieve $0.1 \mathrm{~g}$ chocolate pieces (FoodFirst LTD Auckland, NZ) and adapt to doors. Rats were also habituated to the runway apparatus. In the runway, rats were shaped to nose poke a $2 \mathrm{~cm}$ circular white plastic cap at 
the centre of a $6.5 \mathrm{~cm} \times 6 \mathrm{~cm}$ thin $(8 \mathrm{~mm})$ odourised sponge in a clear Perspex receptacle and to push an object hinged at the rear of its base in order to reveal a food well (recess within a $5 \mathrm{x}$ $9 \times 3 \mathrm{~cm}$ wooden block with inaccessible food beneath) that contained chocolate pieces. Following surgery recovery rats were re-familiarised to both the RAM and the runway.

\section{Spatial working memory in the radial arm maze}

Spatial working memory was tested using the RAM located in the centre of a windowless room $(3 \times 3 \mathrm{~m})$. The maze had a grey-painted wooden $35 \mathrm{~cm}$ wide central hub with 8 aluminium arms (65 cm long x $8.6 \mathrm{~cm}$ wide, with $4.5 \mathrm{~cm}$-high borders), raised $67.5 \mathrm{~cm}$ above the room floor. Clear Perspex walls (19 cm long x $25 \mathrm{~cm}$ high) extended along one side of each arm from the hub to prevent rats from jumping between arms. A black wooden food well $(5 \mathrm{~cm} \times 8.5 \mathrm{~cm} \times 3 \mathrm{~cm})$, with inaccessible food underneath, was placed at the end of each arm. During testing, $2 \times 0.1 \mathrm{~g}$ chocolate pieces were present in each food well at the start of each trial. Clear Perspex guillotine doors, which could be raised up from beneath the hub by a pulley system, controlled access to the hub and arms.

After re-familiarisation post-surgery, the rats received 10 consecutive days of formal spatial memory testing in the RAM. The rat was placed in the central hub and $\sim 10$ seconds later all eight arms were opened, and the rat allowed to make a choice defined as both hind legs over an arm threshold. Once the rat had entered an arm, all the doors were closed and the rat was confined to the arm for $\sim 15$ seconds irrespective of whether it made a correct choice. The rat was then allowed back into the central hub, held in there for $\sim 10$ seconds before all the doors were again opened to allow another arm choice. The trial concluded when the rat had visited all 8 arms, 20 arm choices had been made or 10 minutes had elapsed. 


\section{Non-spatial tasks: Simple discrimination and paired-associate learning}

Both simple discrimination and paired-associate tasks used the same runway apparatus placed on a table, Figure $1 \mathbf{b}$ and $1 \mathbf{c}$. The red Perspex runway $(93 \times 26$ x $26 \mathrm{~cm})$ could be divided by three vertically removable doors (red Perspex, $26 \times 26 \mathrm{~cm}$ ) that were selected on the basis of the specific behavioural task. As for familiarisation, the receptacle was attached $10 \mathrm{~cm}$ from the base of a door and held a thin sponge with the cap at its centre, which always held a food reward (Figure 1d). A different door plus sponge was used for each odour using $20 \mu 1$ Essential Oils of New Zealand in 5ml sunflower oil; the odours were lemon, clove, cinnamon and lime. Light and visually distinct objects (Figure 1e) were attached by hinge at the rear of its base to the top of the black wooden food well, so that a rat could easily push the object back to inspect the well for food reward. A wooden frame (1.8m high x $1.3 \mathrm{~m}$ wide), draped in black fabric enclosed all sides and the top of the apparatus to reduce spatial cues during testing.

The simple discriminations (odour only; object only) and paired-associate tasks used a 'go/no-go' procedure. The first trial for each session began by restraining the rat in the start area for 120 seconds to reacclimatise to the maze; subsequent trials used 20 seconds in this start area. All rats received 12 massed trials per daily session, with 6 go (rewarded) and 6 nogo (non-rewarded) trials in a pseudo-randomised order. No more than three consecutive go or three no-go trials were run consecutively in a session. No identical pair of specific stimuli was used consecutively in the paired-associate task. Correct items or stimulus pairs in the simple discrimination and paired-associate tasks were counterbalanced across rats. For odours, a response was defined as a nose poke into the cap in the centre of the sponge to eat the reward; sniffing the sponge but ignoring the food reward was considered a no-go response for the simple discrimination. Food was always present in the cup at the centre of the sponge 
for the odour-object paired-associate task; the go / no-go aspect concerned the responding to the object at the end of the runway. For objects, a response was defined as any push with nose or paw that tilted the object backwards. A trial was designated as "correct" if the rat responded in less than 8 seconds on go trials or did not respond before 8 seconds in no-go trials.

\section{Simple discrimination}

Half of the rats received training in the simple odour discrimination task followed by the simple object discrimination and vice versa for the other half. Latency between Door X (Figure 1a) and interaction with the odour or object at the end of the runway was recorded. Rats were trained on the simple odour and simple object discrimination task until they reached criterion ( $80 \%$ correct over 2 consecutive days).

For the simple odour discrimination task, a single odour was presented on each trial. The rat had to discriminate which of two odours was paired with a food reward presented in the sponge at the end of Compartment B (Figure 1a). In the simple object discrimination task, a single object was presented on each trial at the end of Compartment B. The rat had to learn which of two objects was paired with food reward presented under the object.

\section{Paired-associate tasks (trace and no-trace)}

These tasks used a new layout of the vertically removable doors; see Fig. 1b and 1c. The rats learned which of two object pairings would be rewarded (e.g. odour $1+$ object A; and odour $2+$ object B) and which two pairings were not rewarded (i.e. odour $1+$ object B; and odour 2 + object A). Irrespective of the pairing, rats always received a reward on presentation of the odour when allowed into the second compartment of the runway. This was compartment $\mathrm{C}$ 
for the "no-trace" condition, in which compartment A was not used; and compartment B was the start compartment (see Figure 1b). The odour was at the end of compartment B for the "trace" condition, for which compartment A was used as the start box (see Figure 1c). Rats in the trace condition were subjected to a 10 second delay between presentations of the odour and object stimuli, by being held in compartment $\mathrm{C}$, whereas rats in the no-trace condition were exposed to the object immediately following their interaction with odour (after eating the reward). In both trace and no-trace conditions, we recorded latency from the time Door Z was lifted until the rat interacted with the object, or refrained from interacting with it for 8 seconds. Different groups of rats were trained on the odour-object and odour-trace-object paired-associate task until they reached criterion ( $80 \%$ correct trials across 3 days) or for a maximum of 50 days.

\section{Post-acquisition recall test}

Recall of the paired-associate task for each group on the trace or no-trace condition was conducted 5 days after the last criterion day. This session used 12 massed trials on a single day in identical fashion as used previously in training for that specific rat.

\section{Histology}

\section{Perfusion and tissue collection}

On the 3 days prior to the recall test, single rats were familiarised to an empty clean cage for 90 minutes in a dark quiet room. Immediately following the last trial in the recall test, the rat was placed in its cage in the familiar dark quiet room 90 minutes prior to perfusion. Rats were deeply anaesthetised with sodium pentobarbital $(125 \mathrm{mg} / \mathrm{kg})$ and perfused transcardially with saline followed by paraformaldehyde (PFA $4 \%$ in $0.1 \mathrm{M}$ phosphate buffer; PB) to fix the brain. Brains were post-fixed in PFA $4 \%$ followed by a minimum of 48 hours in a long-term 
solution ( $20 \%$ glycerol in $0.1 \mathrm{M} \mathrm{PB})$. Coronal $40 \mu \mathrm{m}$ sections were collected using a freezing microtome (Thermofisher, UK) and stored in a cryo-protectant solution (30\% glycerol, 30\% ethylene glycol in $0.1 \mathrm{M} \mathrm{PB}$ ) at $-20^{\circ} \mathrm{C}$ until processed for immunohistochemistry. The coronal sections were collection in two separate series. The first series captured consecutive sections in 5 cryovials for immunohistochemistry. This included sections from the prefrontal cortex to the septal area (approximately +4.7 to $-0.6 \mathrm{~mm}$ from Bregma) and posterior from the mediodorsal thalamus (approximately $-3.5 \mathrm{~mm}$ from Bregma). The second series captured regions for lesion verification. These consecutive sections were collected in 4 cryovials from immediately anterior to the ATN to the posterior mediodorsal thalamus (approximately -0.6 to $-3.5 \mathrm{~mm}$ from Bregma).

\section{Neu-N staining and ATN lesion verification}

Free floating sections through the ATN were washed $(3 \mathrm{X} 10 \mathrm{~min})$ in $0.1 \mathrm{M}$ phosphate buffered saline with Triton-X (0.2\%; PBSTx) before being incubated in endogenous peroxidase blocking buffer for 30 minutes $\left(1 \%\right.$ hydrogen peroxide $\left(\mathrm{H}_{2} \mathrm{O}_{2}\right), 50 \%$ methanol $\left(\mathrm{CH}_{3} \mathrm{OH}\right)$ in $2 \%$ PBSTx). Sections were incubated overnight at $4{ }^{\circ} \mathrm{C}$ in anti-NeuN primary antibody (1:5000; monoclonal-Mouse Cat\# MAB377; Millipore, California USA) in PBSTx with $1 \%$ normal goat serum (NGS; Life Technologies, NZ). Excess antibody buffer was removed with PBSTx and followed by incubation in biotinylated goat anti-mouse secondary antibody (1:1000 Cat\# BP-9200-50: Vector Laboratories, California USA) overnight at $4^{\circ} \mathrm{C}$ in PBSTx and 1\% NGS. Sections were incubated in ExtrAvidin (peroxidase conjugated; 1:1000; Sigma, NSW Australia), PBSTx and 1\% NGS for 2 hours at room temperature. To remove excess ExtrAvidin and Triton X-100, the sections were washed in PBS, in PB, and then in Tris buffer ( $\mathrm{pH} 7.4$ in distilled $\mathrm{H}_{2} 0$ ), to prepare the sections for visualisation with freshly prepared diaminobenzidine (DAB 0.05\%; Sigma, in $0.01 \% \mathrm{H}_{2} \mathrm{O}_{2}$ in Tris buffer). The 
DAB reaction (approximately $5 \mathrm{~min}$ ) was stopped using Tris buffer (1 X $10 \mathrm{~min}$ wash) and sections were placed in $\mathrm{PB}$ at $4^{\circ} \mathrm{C}$ overnight before mounting onto gelatinised slides and allowed to dry. The slides were dehydrated through graded alcohol (70-100\%) before being cleared in xylene and mounted with DPX (06522; Sigma Aldrich) and a coverslip.

The number of Neu-N positive cells in the ATN was used to determine lesion extent. First, the average area showing ATN neurons was outlined and measured in sham rats. This used sections from both the left and right hemisphere for every one in every four $40 \mu \mathrm{m}$ sections throughout the ATN and the area quantified using Image (NIH, USA). NeuNpositive cell staining was photographed at 5x objective on a Leica DM6 B upright microscope and DFC7000T camera (Leica Microsystems, Germany). Automated counts of the cells were obtained through ImageJ (image analysis software, National Institute of Health, NIH, USA). The region of interest was selected and the images were converted to 8-bit grey scale, background was subtracted (rolling $=40$ ), converted to mask and the watershed function was applied, and all neuronal cells above threshold ('MaxEntropy' threshold, circularity $0.5-1.0$ ) were counted. The detection threshold was the same for all sections. Automated NeuN (Neuronal Nuclei) cells counts were made in each sham rat to express cell counts per $\mathrm{mm}^{2}$ in the ATN to derive the average cell count in Sham rats. To accommodate shrinkage of the tissue due to the lesion, NeuN cells remaining in the ATN region taken for each rat with a lesion and this count was then expressed relative to average count of NeuN cells observed in the sham rats. This generated the number of cells spared in each ATN lesion rat $\left(\right.$ cells $\left./ \mathrm{mm}^{2}\right)$. Acceptable lesions were defined as reaching the criterion of $50 \%$ damage to the ATN, with a minimum of $25 \%$ per hemisphere, consistent with previous studies (Mitchell \& Dalrymple-Alford, 2005, 2006; Perry, Mercer, Barnett, Lee, \& Dalrymple-Alford, 2018). 


\section{Zif268 immunohistochemistry}

Free floating sections were stained similar to the NeuN staining protocol, but incubated in rabbit polyclonal Zif268 primary antibody (Egr-1; 1:1000 Cat\# sc-110; Santa Cruz Biotechnology, USA) for 72 hours at $4^{\circ} \mathrm{C}$ in PBSTx with $1 \%$ NGS, followed by incubation in biotinylated goat anti-rabbit secondary antibody (1:1000: Vector Laboratories BA-1000) overnight in PBSTx and 1\% NGS. Following DAB visualisation (approx. 18 minutes), Zif268 positive cell staining in each region of interest was photographed with a 10x objective with a light microscope (Leica, Germany). Automated counts of the cells were obtained through ImageJ (NIH, USA) in the same manner as NeuN-positive cells, except circularity was set at 0.65-1.0. Counts of Zif268-positive cells for all regions used the same threshold algorithm, with between two to six sections per region of interest in each rat quantified. The average Zif268 positive cell count per $\mathrm{mm}^{2}$ across sections (from both hemispheres) within a region of interest was used.

\section{Data analysis}

Statistical analyses were conducted using Statistica (v13; Dell Inc.). A reciprocal transformation of latency data in individual trials was used to ensure homogeneity of variance. Mean latency differences of daily trials (go minus no-go trial latencies) were used to assess discrimination between the different stimuli (simple discrimination) or pairings of stimuli (paired-associate tasks). That is, from the 12 trials on any given testing day, the transformed latencies generated one mean latency difference score from the average of go trials minus the average of the no-go trials. Repeated measures ANOVA, one-way ANOVA and T-tests were conducted to test group difference in task acquisition, trials to criterion and Zif268 counts within and between regions of interest. When significant interactions were 
found, they were further explored through simple main effects analysis. Significance was set at $p<0.05$.

\section{Results}

\section{Lesion verification}

All but one of the 18 rats with ATN lesions met the a priori criterion. The median bilateral damage was $75.5 \%$ (range 61.2-93.5\%, Figure 2). The excluded rat had a satisfactory lesion in one hemisphere only ( $47.9 \%$ total, $75 \%$ left, $20 \%$ right). NeuN count throughout the ATN of intact rats was similar to that reported by Frost et al. (2020); ATN lesion rats showed substantially fewer neuronal cells (Figure 2d). Overall, there was relatively little damage to the mediodorsal thalamus (MD) and reuniens/rhomboid ( $\mathrm{Re} / \mathrm{Rh})$ nuclei. Adjacent to the ATN, MD damage extended approximately from +1.72 to $+2.10 \mathrm{~mm}$ from Bregma, with injury ranging from $0-40.6 \%$ (median=20.9\%); note, the posterior regions of the MD were intact in all cases. Damage to the Re/Rh nuclei, that is, only adjacent to the ATN region and not more posterior, extended approximately -1.08 to- $2.04 \mathrm{~mm}$ from Bregma with injury ranging from $0-32.97 \%$ (median $=6.41 \%)$.

\section{Spatial working memory in the RAM}

As expected, rats with ATN lesions showed severely impaired performance when spatial working memory was tested in the RAM (Figure $3 \mathbf{a}$ and $3 \mathbf{b}$ ). The initial similarity in performance between the groups is because most rats ran for 10 minutes but made relatively few arm entries at the start of testing. The number of errors made by the ATN-lesion and sham-lesion groups diverged on day two of training after which the ATN-lesion group showed no evidence of improvement (Group main effect, $F_{1,29}=122.65, p<0.001$; Group x Day interaction, $\mathrm{F}_{9,261}=11.72$, $\left.\mathrm{p}<0.001\right)$. ATN-lesion rats also made few correct arm choices 
before the first error, whereas the sham-lesion group progressively entered more arms before making an error as testing continued (Group main effect, $F_{1,29}=75.95, p<0.001$; Day $\mathrm{F}_{9,261}=1.21, \mathrm{p}=0.28$; Group $\mathrm{x}$ Day interaction, $\mathrm{F}_{9,261}=4.61$, $\left.\mathrm{p}<0.001\right)$.

\section{Non-spatial simple discrimination tasks}

Both Sham and ATN-lesion groups rapidly acquired the simple odour discrimination task (Figure 4a; Group main effect, $\mathrm{F}_{1,29}=0.06, \mathrm{p}=0.80$; Group x Day interaction, $\mathrm{F}_{5,145}=0.43$, $\mathrm{p}=0.82$ ). Both groups took an average of 4-5 days to reach criterion on the odour discrimination (Sham, mean=4.64, $\mathrm{SD}=0.84 ; \mathrm{ATN}$, mean=4.35, $\mathrm{SD}=0.93 ; \mathrm{t}=0.90, \mathrm{p}=0.37$ ). Although more variable across acquisition, the two groups also learned the simple object discrimination task without any significant differences (Figure 4b; Group main effect, $\mathrm{F}_{1,29}=1.03$, $\mathrm{p}=0.31$; Group $\mathrm{x}$ Day interaction, $\left.\mathrm{F}_{6,174}=1.12, \mathrm{p}=0.34\right)$. Rats took an average of 56 days to reach criterion on the simple object discrimination task (Sham, mean $=5.57$, $\mathrm{SD}=0.85 ; \mathrm{ATN}$, mean=5.29, $\mathrm{SD}=1.04 ; \mathrm{t}=0.79, \mathrm{p}=0.43)$.

\section{Non-spatial paired-associate tasks}

\section{Acquisition}

ATN lesions completely abolished acquisition on both the trace and no-trace conditions of the paired-associate task (Figure 5a, Lesion main effect for difference latency scores, $\left.\mathrm{F}_{1,27}=151.24, \mathrm{p}<0.001\right)$. Despite faster acquisition scores for sham-lesion rats in the trace condition versus sham rats in the no-trace condition, and equally impaired performance in the two ATN-lesion groups, there were no significant interactions (Trace Condition main effect, $\mathrm{F}_{1,27}=5.78, \mathrm{p}=0.02$; Trace Condition $\mathrm{x}$ Lesion $\mathrm{F}_{1,27}=3.51, \mathrm{p}=0.07$; Trace Condition $\mathrm{x}$ Lesion $\mathrm{x}$ Block $\left.\mathrm{F}_{9,243}<0, \mathrm{p}=0.28\right)$. Sham-lesion rats in the trace condition showed greater latency 
differences in the last two blocks of training compared to sham-lesion rats in the no-trace condition (simple main effect of Block 9 and 10, $\mathrm{F}_{1,27}=17.49, \mathrm{P}<0.001$ ).

None of the ATN-lesion rats showed evidence of beginning to acquire the tasks, whereas only two sham-lesion rats in the trace condition and two sham-lesion rats in the notrace condition did not reach criterion on the paired-associate task (85\% correct over three consecutive days). These four sham-lesion rats, however, did show clear evidence of learning based on their latency score on the final block of training (reciprocal mean latency difference for these four rats: $-0.55,-0.55,-0.53,-0.35)$. At this point their latency scores were comparable to the average of those sham-lesion rats in both trace conditions who had reached criterion (reciprocal mean latency difference $=-0.52$ ). One ATN-lesion rat in the trace condition showed some transient, mild evidence of discrimination across Block 8 of training (reciprocal mean latency difference -0.2). For this ATN-lesion rat, however, performance declined by Block 10 of training (reciprocal mean latency difference -0.02), which was the comparable to the average mean latency difference for ATN-lesion rats across both trace conditions (Block 10 reciprocal mean latency difference -0.02).

Rats in all conditions showed an increase in speed of responding over time for rewarded pairings (Figure $5 \mathbf{b}$; main effect of Block $F_{9,243}=75.78, p<0.001$ ). Sham-lesion rats responded faster to rewarded pairings (Lesion main effect $\mathrm{F}_{1,27}=10.21, \mathrm{p}<0.001$ ). Rats in the trace condition, irrespective of lesion, responded faster than rats in the no-trace condition (main effect of Trace Condition $\mathrm{F}_{1,27}=6.77, \mathrm{p}=0.01$; Trace Condition $\mathrm{x}$ Block interaction, $\mathrm{F}_{9,243}=0.43, \mathrm{p}=0.91$; Trace Condition $\mathrm{x}$ Lesion $\left.\mathrm{F}_{1,27}=0.71, \mathrm{p}=0.4\right)$. More rapid acquisition on the paired-associate task by sham-lesion rats in the trace condition may reflect faster response 
latencies for rewarded pairings (simple main effect: Sham, Trace x No-Trace, $F_{1,27}=5.42$, $\mathrm{p}=0.02 ;$ ATN, Trace $x$ No-Trace, $\left.\mathrm{F}_{1,27}=1.70, \mathrm{p}=0.20\right)$.

For non-rewarded pairings, ATN-lesion rats again showed increasing response latencies but now the sham-lesion rats in both conditions slowed their response latencies over time (Figure 5c; non-rewarded pairings: Lesion $\mathrm{F}_{1,27}=65.25$, $\mathrm{p}<0.001$; Lesion $\mathrm{x}$ Block $\left.\mathrm{F}_{9,243}=76.91, \mathrm{p}<0.001\right)$. There were no effects of Trace condition $\left(\mathrm{F}_{1,27}=0.95, \mathrm{p}=0.33\right)$ or Lesion by Trace Condition interaction $\left(\mathrm{F}_{1,27}=0.59, \mathrm{p}=0.44\right)$ for responding to non-rewarded pairings.

\section{Paired-associate task: Five-day recall test}

Sham-lesion rats in both trace conditions retained good discrimination at the 5-day recall test and were thus clearly different to the ATN-lesion rats (Figure 5a; Lesion main effect, $\left.\mathrm{F}_{1,27}=268.27, \mathrm{p}<0.001\right)$. Sham-lesion rats in the trace condition continued to show better performance compared to sham rats in the no-trace condition (Trace Condition main effect, $\mathrm{F}_{1,27}=6.19, \mathrm{p}=0.01$; Lesion $\mathrm{x}$ Trace Condition interaction, $\mathrm{F}_{1,27}=5.76, \mathrm{p}=0.02$; sham groups only, Trace $x$ No-Trace, $F_{1,27}=10.91, p=0.002$ ). Performance for all groups was the same at 5day recall relative to their performance at the end of the training blocks (Block main effect, $F_{1,27}=0.08, p=0.76$; Block $x$ Trace Condition, $F_{1,27}=0.02, p=0.88$; Block $x$ Lesion, $F_{1,27}=0.10$, $\mathrm{p}=0.74$; Block $x$ Trace Condition $x$ Lesion, $\mathrm{F}_{1,27}=0.53$, $\mathrm{p}=0.47$ ).

\section{Zif268 Expression}

\section{Prefrontal regions}

There was higher Zif268 expression across the layers of the prelimbic prefrontal (A32V) region for rats tested in the trace condition (Figure $6 \mathbf{a}$ and $6 \mathbf{b}$, Trace Condition main effect 
$\left.\mathrm{F}_{1,27}=4.43, \mathrm{p}=0.04\right)$. ATN lesions substantially reduced Zif268 expression across these layers (Lesion main effect, $\mathrm{F}_{1,27}=12.08, \mathrm{p}=0.001$ ). There was no interaction of Lesion, Trace Condition or Layer (Lesion $\mathrm{x}$ Trace Condition, $\mathrm{F}_{1,27}=0.46$, $\mathrm{p}=0.5$; Layer $\mathrm{x}$ Trace Condition, $\mathrm{F}_{3,81}=1.6, \mathrm{p}=0.19 ;$ Layer $\mathrm{x}$ Lesion, $\mathrm{F}_{3,81}=0.05, \mathrm{p}=0.98$; Layer $\mathrm{x}$ Lesion $\mathrm{x}$ Trace Condition, $\left.\mathrm{F}_{3,81}=1.8, \mathrm{p}=0.14\right)$.

Despite rats in the trace condition showing higher Zif268 counts in some of the layers of the cingulate $\mathrm{Cg} 1$ (A32D and $\mathrm{A} 24 \mathrm{~b}$ ) and $\mathrm{Cg} 2$ (A24a) regions (Figure 6a and 6c), this was not reflected by the Trace Condition main effect $\left(\mathrm{F}_{1,27}=2.05, \mathrm{p}=0.16\right)$. As in A32V, ATN lesions resulted in a marked reduction of Zif268 throughout the layers of the cingulate cortex (Lesion main effect, $\mathrm{F}_{1,27}=35.76, \mathrm{p}<0.001$ ), with the greatest reduction apparent in the superficial layer (Layer II; Layer x Lesion interaction $\mathrm{F}_{2,54}=9.70, \mathrm{p}<0.001$ ). There were no further interactions for Lesion, Trace Condition or Region in the regions of the cingulate cortex $(\mathrm{F}<2, \mathrm{p}>0.1)$.

\section{Hippocampal and parahippocampal regions}

In the dorsal hippocampus, sham-lesion rats in the trace condition showed increased expression of Zif268 in the dorsal CA1 region of the hippocampus (Figure 6a and $6 \mathbf{b}$; Region $\mathrm{x}$ Trace Condition $\mathrm{x}$ Lesion interaction $\mathrm{F}_{3,75}=8.39, \mathrm{p}<0.001$; simple main effects in CA1:

Sham, Trace $x$ No-Trace $F_{1,25}=5.87, p=0.02 ;$ ATN, Trace $x$ No-Trace $F_{1,25}=3.41, p=0.07$;

Trace, Sham x ATN $F_{1,25}=20.76, p<0.001$; No-Trace, Sham x ATN, $\left.F_{1,25}=0.03, p=0.84\right)$.

There was no Trace Condition effect across other dorsal hippocampal regions (CA3, DG and DG Hilus; Trace Condition main effect $\left(\mathrm{F}_{1,25}=0.6, \mathrm{p}=0.44\right.$; Region $\mathrm{x}$ Trace Condition interaction, $\mathrm{F}_{3,75}=0.4, \mathrm{p}=0.75$ ). ATN-lesions reduced Zif268 expression across the dorsal hippocampus (CA1, CA3, DG and DG Hilus), with ATN-lesion rats in the trace condition 
expressing the lowest Zif268 count (Lesion main effect $\mathrm{F}_{1,25}=7.82, \mathrm{p}=0.009$; Lesion $\mathrm{x}$ Trace Condition interaction $\mathrm{F}_{1,25}=8.62, \mathrm{p}=0.007$; simple main effects of Lesion, ATN, Trace $\mathrm{x}$ NoTrace $F_{1,25}=2.81, p=0.10$; Sham, Trace $x$ No-Trace $F_{1,25}=5.89, p=0.02$; Trace, Sham $x$ ATN $\mathrm{F}_{1,25}=16.85, \mathrm{p}<0.001 ;$ No-Trace, Sham $x$ ATN, $\left.\mathrm{F}_{1,25}<0.01, \mathrm{p}=0.92\right)$.

Despite a drop in expression following ATN-lesions in ventral CA1 (Figure 7a and 7c), there was no Lesion main effect across the ventral CA1 / CA3 hippocampal regions (vCA1 and vCA3; Lesion main effect, $\mathrm{F}_{1,26}=2.55, \mathrm{p}=0.12$ ). There was no Trace Condition effect or any interactions for vCA1 or vCA3 Regions with Trace Condition or Lesion $\left(\mathrm{F}_{1,26}<1.50, \mathrm{p}>0.10\right)$.

ATN-lesion rats presented lower counts of Zif268 in both the dorsal and the ventral subiculum (dSub and vSub; Figure 7a and 7c; sham-lesion M=653.94, ATN-lesion M=538). However, this Lesion main effect in the $\mathrm{dSub}$ and vSub regions did not reach significance (Lesion main effect, $\mathrm{F}_{1,27}=3.96, \mathrm{p}=0.05$ ). There was no Trace Condition effect or any interactions between dSub and vSub Region with Trace Condition or Lesion $\left(\mathrm{F}_{1,27}<0.50\right.$, $\mathrm{p}>0.50)$.

Rats in the trace condition expressed higher mean counts of Zif268 in the perirhinal cortex, but this difference did not reach significance (Figure 7e; Trace Condition main effect, $\left.\mathrm{F}_{1,27}=2.68, \mathrm{p}=0.11\right)$. There was a significant reduction in Zif268 following ATN lesions in this perirhinal region $\left(\mathrm{F}_{1,27}=6.69, \mathrm{p}=0.01\right)$. In the medial and lateral entorhinal regions of the parahippocampal cortex, sham-lesion rats in the no-trace condition showed higher Zif268 expression. This, however, did not result in any significant main effects or interactions (main effect of Trace Condition $\mathrm{F}_{1,27}=0.17, \mathrm{p}=0.67$; Lesion $\mathrm{F}_{1,27}=1.03, \mathrm{p}=0.31$; Trace Condition $\mathrm{x}$ 
Lesion interaction $\mathrm{F}_{1,27}=1.32, \mathrm{p}=0.26$; Region $\mathrm{x}$ Trace Condition $\mathrm{x}$ Lesion interaction $\left.\mathrm{F}_{1,27}=0.41, \mathrm{p}=0.52\right)$

No main effects or interactions of Trace Condition and Lesion were found in the auditory (control) cortex (main effect: Trace Condition, $\mathrm{F}_{1,27}=1.41, \mathrm{p}=0.24$; Lesion, $\mathrm{F}_{1,27}=0.03, \mathrm{p}=0.86$; Trace Condition $\mathrm{x}$ Lesion interaction $\left.\mathrm{F}_{1,27}=2.21, \mathrm{p}=0.14\right)$.

\section{Retrosplenial cortex}

In terms of trace versus no-trace conditions, higher Zif268 counts of were evident in the granular (Rga and $\mathrm{Rgb})$, and dysgranular (Rdg) regions of the retrosplenial cortex for rats in the trace condition, especially in the superficial layers (Figure 8; Trace Condition main effect, $\mathrm{F}_{1,27}=5.26, \mathrm{p}=0.02$, Layer $\mathrm{x}$ Trace Condition interaction, $\mathrm{F}_{1,27}=5.82, \mathrm{p}=0.02$; simple effect of Trace $x$ No-Trace in superficial layer, $\mathrm{F}_{1,27}=5.90, \mathrm{p}=0.02$; deep layer $\mathrm{F}_{1,27}=2.44, \mathrm{p}=0.12$ ). ATN lesions substantially reduced Zif268 positive counts in all retrosplenial regions (Figure 8; main effect of Lesion, $\left.\mathrm{F}_{1,27}=117.98, \mathrm{p}<0.001\right)$. This effect, however, was especially obvious in the superficial layers compared to the deep layers (Layer by Lesion interaction, $\left.\mathrm{F}_{1,27}=114.00, \mathrm{p}<0.001\right)$. There was no Trace Condition by Lesion interaction $\left(\mathrm{F}_{1,27}=0.61\right.$, $\mathrm{p}=0.43)$, Region by Trace Condition interaction $\left(\mathrm{F}_{2,54}=2.03, \mathrm{p}=0.14\right)$, Region $\mathrm{x}$ Trace Condition $\mathrm{x}$ Lesion interaction $\left(\mathrm{F}_{2,54}=0.98, \mathrm{p}=0.39\right)$, or Layer by Trace Condition by Lesion interaction found in the regions of the retrosplenial cortex $\left(\mathrm{F}_{1,27}=1.31, \mathrm{p}=0.26\right)$.

\section{Discussion}

The aim of this study was to examine the effects of ATN lesions on non-spatial odour-object paired-associate tasks. The only previous evidence that ATN lesions affect non-spatial memory were instances when task demands taxed the ability to make temporal 
discriminations among a list of multiple items within a discrete episode (Dumont \& Aggleton, 2013; Wolff, Gibb, \& Dalrymple-Alford, 2006). We anticipated that the effects of ATN lesions would therefore be most prevalent when an explicit temporal delay was imposed between the presentation of the odour and object stimuli used to establish the paired-associate memory. However, none of the rats with ATN lesions showed evidence of acquisition on either the standard odour-object paired associate task (i.e. with no explicit delay) or the odour-trace-object paired associate task (i.e., with a 10-second delay). The ATN-lesion rats were not able to slow or inhibit responding to the non-rewarded odour-object pairings, showing no evidence of learning despite the extended period of training provided. The delay manipulation had impact, however, based on faster running speeds for both sham and lesion groups of rats on the rewarded trials for the trace compared to no-trace paired-associate tasks. The sham-lesion rats in the trace and no-trace conditions did not differ, however, in terms of learning to slow running speeds on non-rewarded trials.

Another example of the impact of the delay manipulation was that the intact rats showed evidence of increased Zif268 expression in dorsal hippocampal CA1 when the 10second delay was used between the presentation of the odour and object cues, relative to the no-trace condition. By contrast, IEG activity in CA1 was relatively reduced in the ATNlesion rats in the trace condition, albeit non-significantly, compared to their respective notrace counterparts. The increased Zif268 response for sham rats when a delay was used reinforces the lesion evidence for importance of the CA1 neurons for temporal memory functions (Kesner, Hunsaker, \& Gilbert, 2005). There was also evidence in both sham-lesion rats and ATN-lesion rats, trained with the delay, of increased Zif268 expression in the superficial regions of the prefrontal cortex and the superficial regions of the retrosplenial cortex. These effects were found despite the ATN lesions strongly reducing Zif268 activity in 
these cortical regions. It is, however, impossible to know whether this increased cortical IEG expression reflected the presence of a delay per se or the use of a delay in the context of the specific paired-associate task.

The poor performance on these paired-associate memory tasks by the ATN-lesion rats does not appear to be due to poor inhibition, impaired sensory processing, or a simple failure to attend to the stimuli, per se. This was shown by their rapid acquisition when learning both the simple object discrimination and the simple odour discrimination, which was equal to that shown by sham-lesion rats. The task demands for these simple discriminations were identical to the paired-associate task and used the same apparatus and go/no-go requirements. The rapid acquisition of the simple discrimination tasks in the runway contrasted with slow, albeit unimpaired acquisition in rats in our laboratory of even simple odour and, especially, simple object discriminations when trained on the cheeseboard (Bell, 2007). It is possible that the use of a runway and the explicit reduction of distracting spatial cues, plus the need to actively interact with the object to determine the presence of food reward, facilitated attention to the non-spatial stimuli in the current study. Given the severity of the impairment in this nonspatial paired-associate task in the runway, together with the expected deficit in spatial working memory when tested on the RAM, we conclude that higher order declarative memory processes often appear remarkably reliant on the integrity of the ATN. More fundamental learning and memory processes are not dependent on the ATN, and this includes the acquisition of relatively simple spatial reference memory discriminations (Gibb, Wolff, \& Dalrymple-Alford, 2006).

The pattern of findings reported here strongly reinforces the idea that ATN constitute a critical node within the hippocampal-diencephalic-cingulate network that supports episodic 
memory (Bubb, Kinnavane, \& Aggleton, 2017). They show that paired-associate memory and related biconditional associations need not depend on whether one component demands attention to distal spatial cues, which contrasts with the conclusions offered by Dumont, Amin, and Aggleton (2014). The reason behind the failure to detect non-spatial biconditional learning deficits when local contextual cues were either visual, thermal or floor texture, as found by Dumont et al. (2014), is not clear. One possibility, based on Kesner and colleagues' theory (Hunsaker \& Kesner, 2018), is that the presence of paired-associate deficits will rely on whether the brain region in question is strongly involved in processing the particular component attributes. This perspective does not, however, immediately lead us to answer why odours and objects would be relevant for processing by ATN neurons, but local contextual cues would not, even if the latter cues are more strongly supported by alternate brain regions. Perhaps the integration of cues within a local context is a less demanding task than the integration of arbitrary non-spatial odour and object cues. Another possibility is that the use in the present study of a consistent reward to ensure that the rats attended to the odour meant that this cue did not carry sufficient salience for rats with ATN lesions once confronted with the anticipation of a subsequent object that was rewarded $50 \%$ of the time. However, selective attention rather than sustained attention may be impaired by ATN lesions (Chudasama, Bussey, \& Muir, 2001; Wright, Vann, Aggleton, \& Nelson, 2015), and there is no reason to suppose that an attentional bias would feature in our task, so it appears that the deficit in paired-associate learning in the current study is something unique to the binding of the elemental cues as a single representation to guide behavioural choices. Whatever the reason behind the impairment produced by ATN lesions in the current study, it is clearly a profound deficit. 
Importantly, our findings provide an additional example that ATN lesions need not always recapitulate deficits produced by lesions to the hippocampus (Sziklas \& Petrides, 2004, 2007). While there is evidence that ATN lesions can cause greater spatial memory impairments than fornix lesions (Warburton \& Aggleton, 1999), or deficits in object-place and geometric discrimination tasks that are not found with fornix lesions (Aggleton, Poirier, Aggleton, Vann, \& Pearce, 2009; Sziklas, Lebel, \& Petrides, 1998), our study provides the first evidence of a memory impairment after ATN lesions that is not found with hippocampal lesions. Based on prior evidence of deficits associated with memory for temporal order and the reduction in CA1 spine density after ATN lesions (Dumont \& Aggleton, 2013; Harland, Collings, McNaughton, Abraham, \& Dalrymple-Alford, 2014; Wolff et al., 2006), and the pattern of impaired non-spatial paired-associate learning after selective hippocampal CA1 lesions, but not CA3 lesions (Gilbert \& Kesner, 2002; Kesner et al., 2005), we anticipated that ATN lesion effects might be particularly severe or even restricted to the instance in which a 10-second delay was imposed between the presentation of the odour and the subsequent object. That is, prior research would predict that deficits after ATN lesions would be most apparent when the non-spatial odour-object paired associate task included an explicit 10-second trace between the stimuli, as this is the only condition in which hippocampal CA1 lesions cause acquisition failure (Kesner et al., 2005). Unlike hippocampal lesions, odourobject paired associate learning without an explicit intervening delay was abolished by ATN lesions, at least within the extended training period used here. There would be a very brief delay between odour and object in the standard ("no delay") task, but if this is sufficient to abolish learning after ATN lesions then the ATN must be critical in maintaining representations in most memory tasks. This explanation seems unlikely given the normal acquisition of both simple object discriminations and more difficult configural object discriminations (Moran \& Dalrymple-Alford, 2003). In addition, the odour and object were 
presented simultaneously in our previous demonstration of impaired object-odour pairedassociate learning after ATN lesions, which used the cheeseboard protocol (Bell, 2007). In that previous study, we found that lesions of the intralaminar thalamic nuclei also delayed acquisition of the object-odour association memory. In the current study, however, there was relatively little to minimal damage to the immediately adjacent intralaminar or mediodorsal thalamic regions. It is an empirical question whether the paired associate deficits revealed by the current study are selective to the ATN; the important point is that selective ATN lesions have a dramatic effect on this task.

Our findings bring the effects of ATN lesions in rats closer into line with pairedassociate memory impairments in clinical amnesia after injury to the mammillary body ATN axis (Rempel-Clower, Zola, Squire, \& Amaral, 1996; Squire et al., 2020). There is, however, a clear difference in the slow acquisition of paired associate memory in intact rats and the rapid acquisition of paired-associate memory in humans with intact memory systems. Paired-associate tasks are assumed to reflect episodic-like memory by measuring the ability to form unique representations of multiple stimuli rather than processing individual components (Eichenbaum \& Fortin, 2009; Crystal \& Smith, 2014). The length of training required (4 to 5 weeks for a total of 600 training trials in intact rats) could be construed as evidence of the task becoming more rule-based or semantic-like. It seems unlikely that rats were able to learn the paired-associate rule by trial and error within each 12-trial session test day, because the four options were presented in a semi-randomised order. It seems more likely that rats were recruiting episodic-like memory of previous trials within a session to guide their responding. Future work could first train intact rats on one or more such nonspatial paired-associate tasks, before testing acquisition of a new task after ATN lesions. In this way, the general rule of making an association would already be acquired and, perhaps, 
the rate of acquisition would then be relatively rapid for a new task in intact rats. In addition, temporary chemogenetic or optogenetic manipulations of the ATN could provide an opportunity to investigate the impact that these nuclei have on retarding acquisition rather than preventing acquisition or on recall rather than acquisition. It would also be informative to learn which of the many neural projections from the ATN support this paired-associate example of non-spatial learning. It may be the case that the impact of ATN lesions on this task involves frontal brain regions and / or the retrosplenial cortex and their involvement in rule-based and knowledge-based systems, rather than event-based memory (Hunsaker \& Kesner, 2018). These issues could also be addressed using contralateral disconnection lesions involving the ATN, an approach that has been used to successfully demonstrate the systemwide influence of the ATN in spatial tasks (Dumont, Petrides, \& Sziklas, 2010; Warburton, Baird, Morgan, Muir, \& Aggleton, 2000, 2001).

The present study provides novel evidence that ATN lesions produce impairments in non-spatial paired-associate learning and memory, irrespective of the presence of an explicit temporal component. Moreover, extensive training demonstrated no evidence of learning in these ATN-lesion rats, establishing a major behavioural deficit that extends the involvement of the ATN beyond both space and time. Evidence from these paired-associate tasks suggests a new role for the ATN within the 'hippocampal-diencephalic-cingulate' memory network (Bubb et al., 2017). The contrast with the effects of hippocampal lesions highlights the ATN as a critical node within the extended memory system. This strengthens the view that the ATN do not operate primarily as a relay for hippocampal information (Wolff \& Vann, 2019). Instead, the ATN may actively control the generation of complex memory representations in the brain. 


\section{Acknowledgements}

This research was supported by University of Canterbury equipment and research grants and Early Career support (JJH) from Brain Research New Zealand - Rangahau Roro Aotearoa.

Competing Interests

None.

Author Contributions

JJH and JCDA: funding, concept and design, writing and editing drafts, statistical analysis and interpretation of data; $\mathrm{JJH}$ : conducting experiments.

\section{Data accessibility}

Will be available on Mendeley

\section{Abbreviations}

$\mathrm{A} 29 \mathrm{a}=$ area $29 \mathrm{a}$ of the granular retrosplenial cortex; $\mathrm{A} 29 \mathrm{~b}=$ area $29 \mathrm{~b}$ of the granular retrosplenial cortex; A29c $=$ area 29c of the granular retrosplenial cortex; A30 $=$ area 30 of the dysgranular retrosplenial cortex; $\mathrm{A} 32 \mathrm{D}=$ area $32 \mathrm{D}$ of the anterior cingulate cortex; $\mathrm{A} 32 \mathrm{~V}$ $=$ area $32 \mathrm{~V}$ of the medial prefrontal cortex; $\mathrm{A} 24 \mathrm{a}=$ area $24 \mathrm{a}$ of the anterior cingulate cortex; $\mathrm{A} 24 \mathrm{~b}=$ area $24 \mathrm{~b}$ of the anterior cingulate cortex $\mathrm{ATN}=$ anterior thalamic nuclei; $\mathrm{AD}=$ anterodorsal thalamic nuclei; $\mathrm{AM}=$ anteromedial thalamic nuclei; $\mathrm{AV}=$ anteroventral thalamic nuclei; $\mathrm{CA} 1=$ cornu ammonis $1 ; \mathrm{CA} 3=$ cornu ammonis $3 ; \mathrm{cc}=$ corpus callosum; $\mathrm{Cg} 1 / \mathrm{Cg} 2=$ cingulate area $1 / 2 ; \mathrm{DG}=$ dentate gyrus; DG Hilus = hilus of the dentate gyrus; $\mathrm{dSub}=$ dorsal subiculum; IEG = immediate early gene; $1 \mathrm{EC}=$ lateral entorhinal cortex $; \mathrm{MD}=$ mediodorsal thalamic nuclei $\mathrm{mEC}=$ medial entorhinal cortex $; \mathrm{MTT}=$ mammillothalamic tract; $\mathrm{NAc}=$ nucleus accumbens; $\mathrm{PRh}=$ perirhinal cortex $; \mathrm{Re} / \mathrm{Rh}=$ reuniens $/$ rhomboid 
nuclei; Rga = dysgranular a retrosplenial cortex; Rgb = dysgranular b retrosplenial cortex; $\mathrm{Rdg}=$ granular retrosplenial cortex $; \mathrm{vCA} 1=$ dorsal cornu ammonis area 1 ; vCA3 = dorsal cornu ammonis area 3; vSub = ventral subiculum.

\section{References}

Aggleton, J. P., Amin, E., Jenkins, T. A., Pearce, J. M., \& Robinson, J. (2011). Lesions in the anterior thalamic nuclei of rats do not disrupt acquisition of stimulus sequence learning. The Quarterly Journal of Experimental Psychology, 64(1), 65-73.

Aggleton, J. P., \& Nelson, A. J. D. (2015). Why do lesions in the rodent anterior thalamic nuclei cause such severe spatial deficits? Neuroscience \& Biobehavioral Reviews. 54, 131144.

Aggleton, J. P., Poirier, G. L., Aggleton, H. S., Vann, S. D., \& Pearce, J. M. (2009). Lesions of the fornix and anterior thalamic nuclei dissociate different aspects of hippocampaldependent spatial learning: implications for the neural basis of scene learning. Behavioral neuroscience, 123(3), 504-519.

Bell, R. (2007). Anterior and lateral thalamic lesions in object-odour paired associate learning (unpublished master's thesis). University of Canterbury, Christchurch, New Zealand.

Bubb, E. J., Kinnavane, L., \& Aggleton, J. P. (2017). Hippocampal-diencephalic-cingulate networks for memory and emotion: An anatomical guide. Brain and Neuroscience Advances, 1(1), 1-20.

Carlesimo, G. A., Lombardi, M. G., \& Caltagirone, C. (2011). Vascular thalamic amnesia: A reappraisal. Neuropsychologia, 49(5), 777-789. 
Chudasama, Y., Bussey, T. J., \& Muir, J. L. (2001). Effects of selective thalamic and prelimbic cortex lesions on two types of visual discrimination and reversal learning. The European Journal of Neuroscience, 14(6), 1009-1020.

Crystal, J. D., \& Smith, A. E. (2014). Binding of episodic memories in the rat. Current Biology, 24(24), 2957-2961.

Dalrymple-Alford, J. C., Harland, B. C., Loukavenko, E. A., Perry, B., Mercer, S., Collings, D. A., Ulrich, K., Abraham, W. C., McNaughton, N., \& Wolff, M. (2015). Anterior thalamic nuclei lesions and recovery of function: Relevance to cognitive thalamus. Neuroscience and Biobehavioural Reviews, 54, 145-160.

Dumont, J. R., \& Aggleton, J. P. (2013). Dissociation of recognition and recency memory judgments after anterior thalamic nuclei lesions in rats. Behavioral Neuroscience, 127(3), $415-431$.

Dumont, J. R., Amin, E., \& Aggleton, J. P. (2014). Selective importance of the rat anterior thalamic nuclei for configural learning involving distal spatial cues. The European Journal of Neuroscience, 39(2), 241-256.

Dumont, J. R., Petrides, M., \& Sziklas, V. (2010). Fornix and retrosplenial contribution to a hippocampo-thalamic circuit underlying conditional learning. Behavioural Brain Research, 209(1), 13-20.

Dupire, A., Kant, P., Mons, N., Marchand, A. R., Coutureau, E., Dalrymple-Alford, J. C., \& Wolff, M. (2013). A role for anterior thalamic nuclei in affective cognition: interaction with environmental conditions. Hippocampus, 23(5), 392-404.

Eichenbaum, H. B., \& Fortin, N. J. (2009). The neurobiology of memory based predictions. Philosophical Transactions of the Royal Society B: Biological Sciences, 364(1521), 11831191. 
Frost, B. E., Cafalchio, M., Martin, S. K., Islam, M. N., Aggleton, J. P., \& O’Mara, S. M. (2020). Spatial Coding in the Subiculum Requires Anterior Thalamic Inputs. bioRxi, doi: https://doi.org/10.1101/2020.01.31.928762

Gibb, S. J., Wolff, M., \& Dalrymple-Alford, J. C. (2006). Odour-place paired-associate learning and limbic thalamus: Comparison of anterior, lateral and medial thalamic lesions. Behavioural Brain Research, 172(1), 155-168.

Gilbert, P. E., \& Kesner, R. P. (2002). Role of rodent hippocampus in paired-associate learning involving associations between a stimulus and a spatial location. Behavioral Neuroscience, 116(1), 63.

Gilbert, P. E., \& Kesner, R. P. (2003). Localization of function within the dorsal hippocampus: the role of the CA3 subregion in paired-associate learning. Behavioral Neuroscience, 117(6), 1385.

Harding, A., Halliday, G., Caine, D., \& Kril, J. (2000). Degeneration of anterior thalamic nuclei differentiates alcoholics with amnesia. Brain: A Journal of Neurology, 123(1), 141154.

Harland, B. C., Collings, D. A., McNaughton, N., Abraham, W. C., \& Dalrymple-Alford, J. C. (2014). Anterior thalamic lesions reduce spine density in both hippocampal CA1 and retrosplenial cortex, but enrichment rescues CA1 spines only. Hippocampus, 24(10), 12321247.

Hunsaker, M. R., \& Kesner, R. P. (2018). Unfolding the cognitive map: The role of hippocampal and extra-hippocampal substrates based on a systems analysis of spatial processing. Neurobiology of Learning and Memory, 147, 90-119.

Hunsaker, M. R., Thorup, J. A., Welch, T., \& Kesner, R. P. (2006). The role of CA3 and CA1 in the acquisition of an object-trace-place paired-associate task. Behavioral Neuroscience, $120(6), 1252$. 
Jenkins, T. A., Dias, R., Amin, E., \& Aggleton, J. P. (2002). Changes in Fos expression in the rat brain after unilateral lesions of the anterior thalamic nuclei. European Journal of Neuroscience, 16(8), 1425-1432.

Jenkins, T. A., Dias, R., Amin, E., Brown, M. W., \& Aggleton, J. P. (2002). Fos imaging reveals that lesions of the anterior thalamic nuclei produce widespread limbic hypoactivity in rats. Journal of Neuroscience, 22(12), 5230-5238.

Jo, Y. S., \& Lee, I. (2010). Disconnection of the hippocampal-perirhinal cortical circuits severely disrupts object-place paired associative memory. Journal of Neuroscience, 30(29), 9850-9858.

Kehoe, E. J. (1988). A layered network model of associative learning: learning to learn and configuration. Psychological Review, 95(4), 411.

Kesner, R. P., Hunsaker, M. R., \& Gilbert, P. E. (2005). The role of CA1 in the acquisition of an object-trace-odor paired associate task. Behavioral Neuroscience, 119(3), 781-786.

Kopelman, M. D. (2015). What does a comparison of the alcoholic Korsakoff syndrome and thalamic infarction tell us about thalamic amnesia? Neuroscience \& Biobehavioral Reviews, 54, 46-56.

Lee, I., \& Solivan, F. (2008). The roles of the medial prefrontal cortex and hippocampus in a spatial paired-association task. Learning \& Memory, 15(5), 357-367.

Loukavenko, E. A., Wolff, M., Poirier, G. L., \& Dalrymple-Alford, J. C. (2015). Impaired spatial working memory after anterior thalamic lesions: recovery with cerebrolysin and enrichment. Brain Structure and Function, 221(4), 1955-1970.

Maillard, A., Laniepce, A., Cabé, N., Boudehent, C., Chételat, G., Urso, L., Eustache, F., Vabret, F., Segobin, S., \& Pitel, A. L. (2021). Temporal Cognitive and Brain Changes in Korsakoff's Syndrome. Neurology, 96(15), e1987-1998 
McDonald, R. J., Murphy, R. A., Guarraci, F. A., Gortler, J. R., White, N. M., \& Baker, A. G. (1997). Systematic comparison of the effects of hippocampal and fornix-fimbria lesions on acquisition of three configural discriminations. Hippocampus, 7(4), 371-388.

Mitchell, A. S., \& Dalrymple-Alford, J. C. (2005). Dissociable memory effects after medial thalamus lesions in the rat. The European Journal of Neuroscience, 22(4), 973-985.

Mitchell, A. S., \& Dalrymple-Alford, J. C. (2006). Lateral and anterior thalamic lesions impair independent memory systems. Learning \& Memory, 13(3), 388-396.

Moran, J. P., \& Dalrymple-Alford, J. C. (2003). Perirhinal Cortex and Anterior Thalamic Lesions: Comparative Effects on Learning and Memory. Behavioral Neuroscience, 117(6), $1326-1341$.

Nelson, A. J. D. (2021). The anterior thalamic nuclei and cognition: a role beyond space? Neuroscience and Biobehavioral Reviews, 126, 1-11

Perry, B. A. L., Mercer, S. A., Barnett, S. C., Lee, J., \& Dalrymple-Alford, J. C. (2018). Anterior thalamic nuclei lesions have a greater impact than mammillothalamic tract lesions on the extended hippocampal system. Hippocampus, 28(2), 121-135.

Rempel-Clower, N. L., Zola, S. M., Squire, L. R., \& Amaral, D. G. (1996). Three cases of enduring memory impairment after bilateral damage limited to the hippocampal formation. Journal of Neuroscience, 16(16), 5233-5255.

Ridley, R. M., Maclean, C. J., Young, F. M., \& Baker, H. F. (2002). Learning impairments in monkeys with combined but not separate excitotoxic lesions of the anterior and mediodorsal thalamic nuclei. Brain Research, 950(1-2), 39-51.

Squire, L. R., Kim, S., Frascino, J. C., Annese, J., Bennett, J., Insausti, R., \& Amaral, D. G. (2020). Neuropsychological and neuropathological observations of a long-studied case of memory impairment. Proceedings of the National Academy of Sciences, 117(47), 2988329893. 
Sweeney-Reed, C. M., Buentjen, L., Voges, J., Schmitt, F. C., Zaehle, T., Kam, J. W. Y., Kaufmann, J., Heinze, H. J., Hinrichs, H., Knight, R. T., \& Rugg, M. D. (2021). The role of the anterior nuclei of the thalamus in human memory processing. Neuroscience and Biobehavioral Reviews, 126, 146-158.

Sziklas, V., Lebel, S., \& Petrides, M. (1998). Conditional associative learning and the hippocampal system. Hippocampus, 8(2), 131-137.

Sziklas, V., \& Petrides, M. (1999). The effects of lesions to the anterior thalamic nuclei on object-place associations in rats. The European Journal of Neuroscience, 11(2), 559-566. Sziklas, V., \& Petrides, M. (2002). Effects of lesions to the hippocampus or the fornix on allocentric conditional associative learning in rats. Hippocampus, 12(4), 543-550.

Sziklas, V., \& Petrides, M. (2004). Egocentric conditional associative learning: Effects of restricted lesions to the hippocampo-mammillo-thalamic pathway. Hippocampus, 14(8), 931934.

Sziklas, V., \& Petrides, M. (2007). Contribution of the anterior thalamic nuclei to conditional learning in rats. Hippocampus, 17(6), 456-461.

Warburton, E. C., \& Aggleton, J. P. (1999). Differential deficits in the Morris water maze following cytotoxic lesions of the anterior thalamus and fornix transection. Behavioural Brain Research, 98(1), 27-38.

Warburton, E. C., Baird, A. L., Morgan, A., Muir, J. L., \& Aggleton, J. P. (2000).

Disconnecting hippocampal projections to the anterior thalamus produces deficits on tests of spatial memory in rats. The European Journal of Neuroscience, 12(5), 1714-1726.

Warburton, E. C., Baird, A. L., Morgan, A., Muir, J. L., \& Aggleton, J. P. (2001). The conjoint importance of the hippocampus and anterior thalamic nuclei for allocentric spatial learning: evidence from a disconnection study in the rat. Journal of Neuroscience, 21(18), 7323-7330. 
Wolff, M., Gibb, S. J., \& Dalrymple-Alford, J. C. (2006). Beyond Spatial Memory: The Anterior Thalamus and Memory for the Temporal Order of a Sequence of Odor Cues. Journal of Neuroscience, 26(11), 2907-2913.

Wolff, M., \& Vann, S. D. (2019). The cognitive thalamus as a gateway to mental representations. Journal of Neuroscience, 39(1), 3-14.

Wright, N. F., Vann, S. D., Aggleton, J. P., \& Nelson, A. J. D. (2015). A Critical Role for the Anterior Thalamus in Directing Attention to Task-Relevant Stimuli. Journal of Neuroscience, 35(14), 5480-5488. 
Figure Captions

(a)

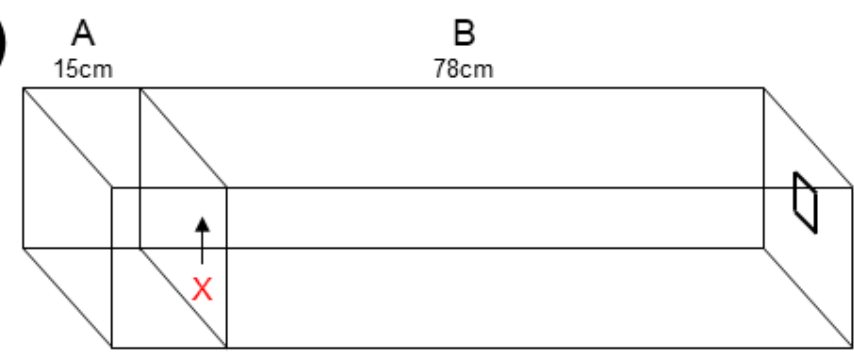

(b)

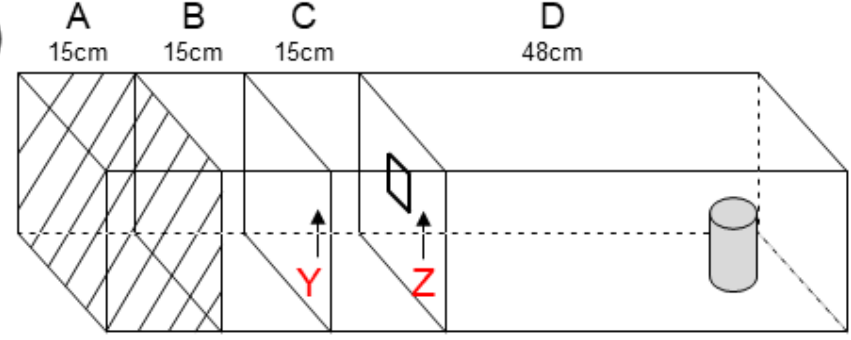

(c)

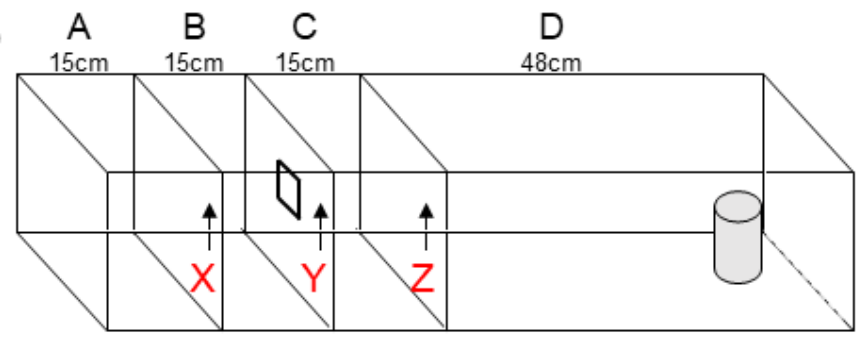

(d)

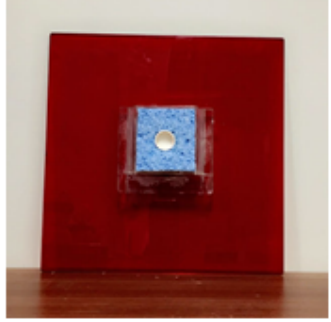

(e)

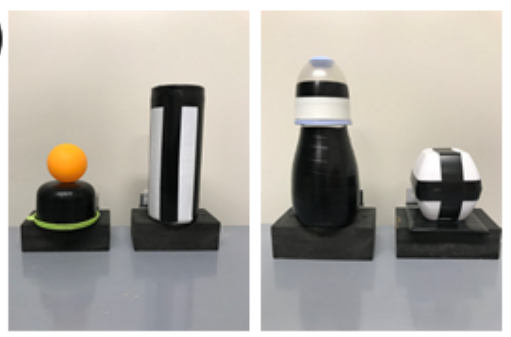

Figure 1. Runway apparatus used in both the simple odour and simple object discrimination tasks, and the odour-object paired associate memory tasks. (a) For simple discriminations, rats were placed in the start box (A), door X was removed and we recorded the latency to interact with the odour (d) or a single object (e) located at the end of compartment B. Runway apparatus for No Trace (b) and Trace (c) paired-associate memory tasks. (b) Rats began the No Trace paired-associate task in Compartment B for 120 seconds on the first trial on any session and for $20 \mathrm{sec}$ on the remaining trials (Compartment A was not used). Door Y was removed to give access to the odour on door $\mathrm{Z}$ at the end of Compartment $\mathrm{C}$, per (d). Immediately after the food reward was consumed from the plastic cap in the centre of the odour sponge, Door $\mathrm{Z}$ was removed and we recorded the latency to interact with a single object, per (e) at the end of Compartment D. (c) Rats began the Trace paired-associate memory task in Compartment A for 120 seconds on the first trial per day and 20 secs on 
remaining trials. Door X was removed to give access to the odour sponge, per (d), on door Y at the end of Compartment B. Door Y was immediately removed on eating the food and the rat was retained for a 10-second trace (delay in Compartment C) before Door Z was removed. We recorded latency from removal of Door $\mathrm{Z}$ to interaction with the object at the end of Compartment D. 
(a)
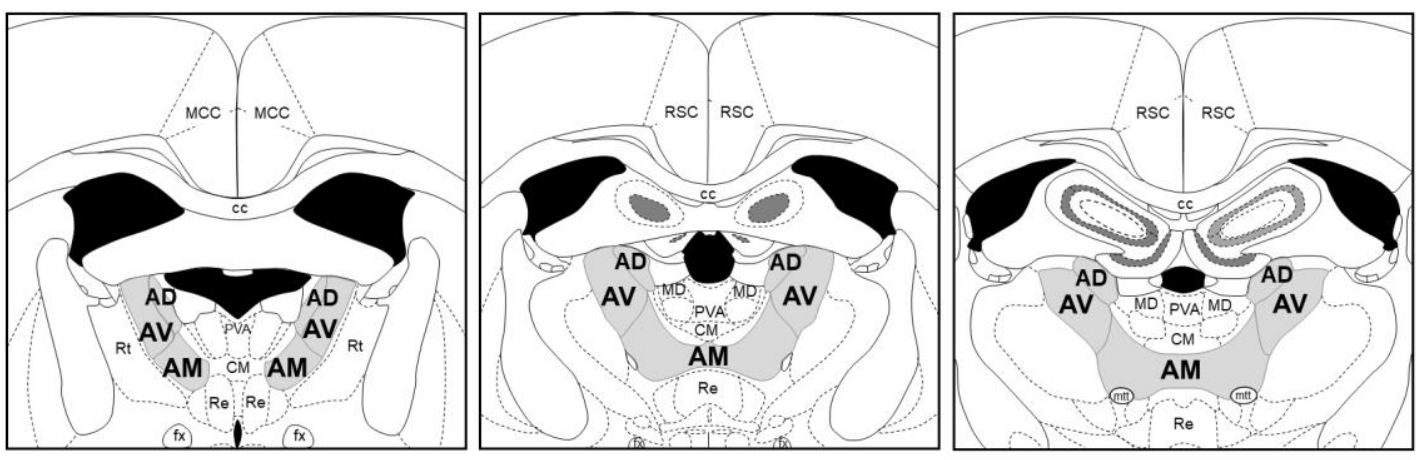

(b)
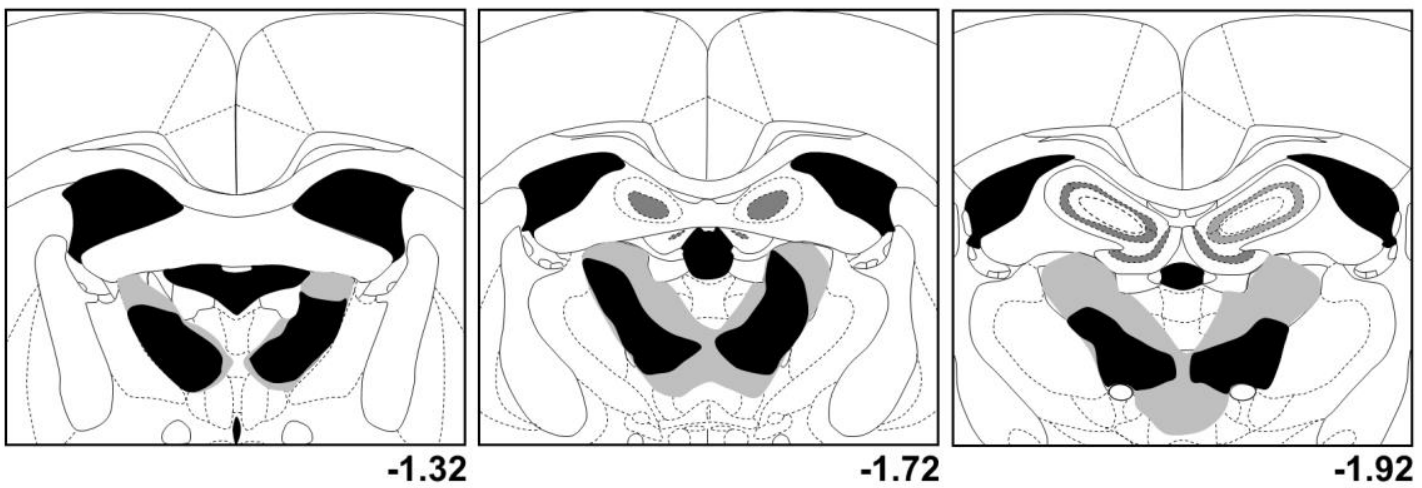

(c)

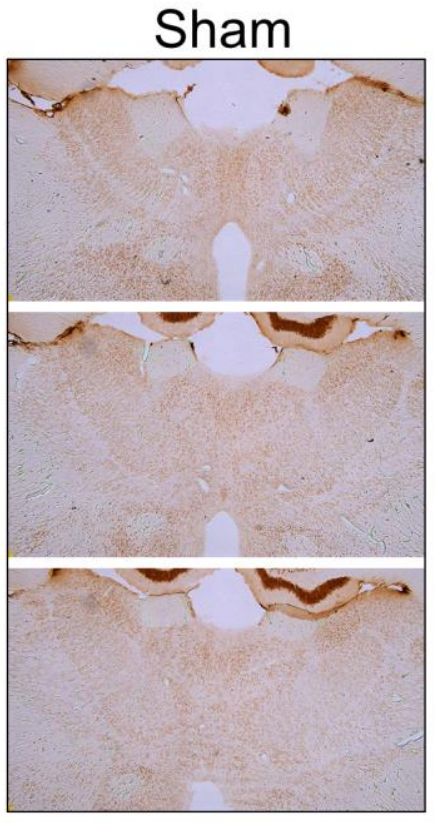

(d)
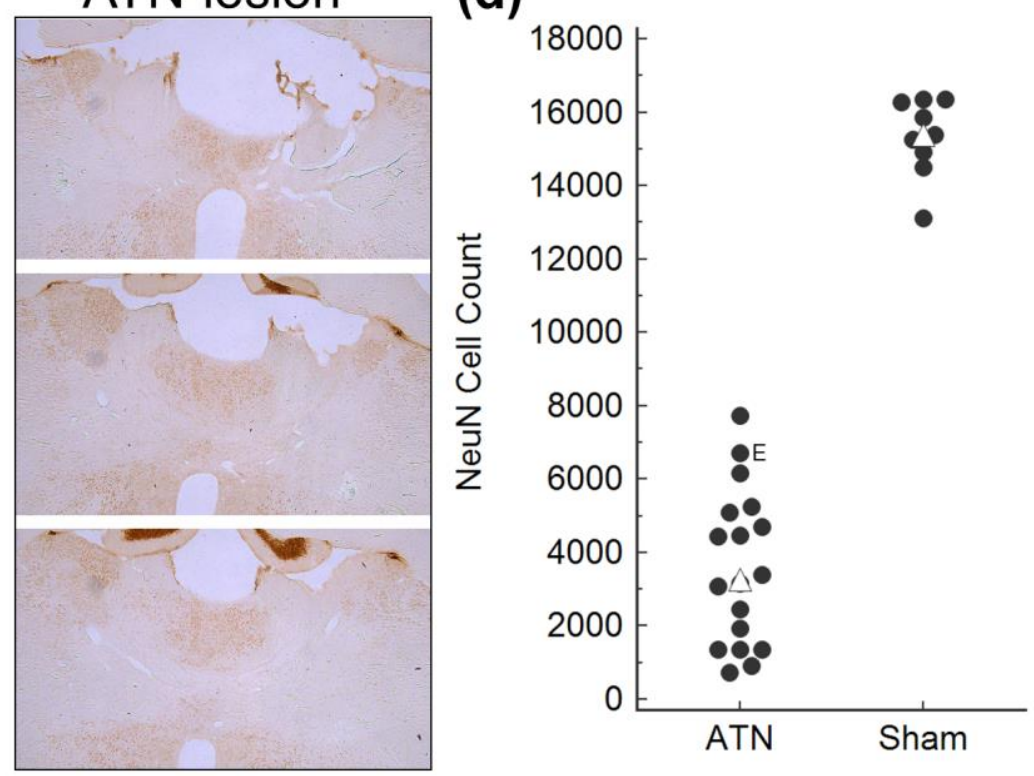

Figure 2. (a) Schematic diagrams indicating the location of the anterodorsal (AD),

anteroventral (AV) and anteromedial (AM) subregions of the anterior thalamic nuclei (ATN).

(b) Schematic diagrams of coronal sections through the anterior thalamus representing the

largest (grey) and smallest (black) ATN lesions across the anterior thalamus. (c) $2.5 \mathrm{x}$

magnification photomicrographs of $\mathrm{NeuN}$ staining in a sham rat and an ATN-lesion with an average lesion. Compared to sham, there is clear reduction of cells as well as collapse in the 
tissue although AD and dorsal AV showed some sparing. (d) The number of ATN neurons (NeuN-positive) was substantially lower in all ATN-lesion rats compared to sham-lesion rats; the count was made from a 1:4 series throughout the ATN and is therefore an underestimate. $\Delta=$ median rat in each lesion group $; \mathrm{E}=$ excluded $\mathrm{ATN}$-lesion rat due less damage than apriori criterion of at least $50 \%$ damage. Sham NeuN count includes four of the trace and five of the no-trace condition rats. $\mathrm{cc}=$ corpus callosum; $\mathrm{CM}=$ central medial thalamic nuclei; $\mathrm{fx}$ $=$ fornix $; \mathrm{MCC}=$ mid cingulate cortex $\mathrm{MD}=$ mediodorsal thalamic nuclei $; \mathrm{mtt}=$ mammillothalamic tract; PVA = paraventricular thalamic nuclei, anterior; $\mathrm{RSC}=$ retrosplenial cortex; $\mathrm{Rt}=$ reticular nucleus . 
(a)

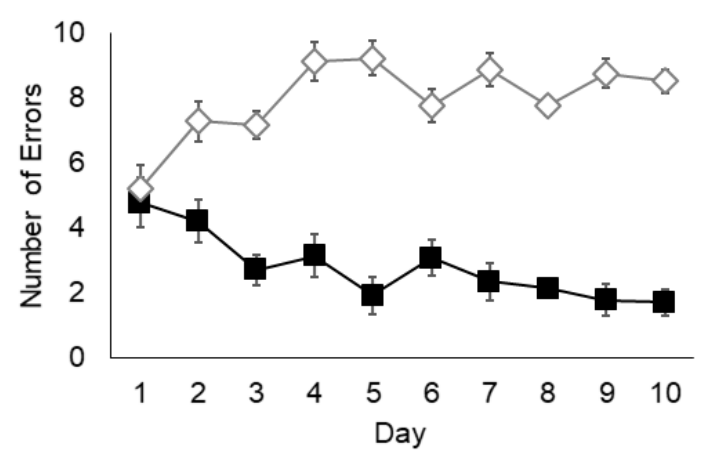

(b)

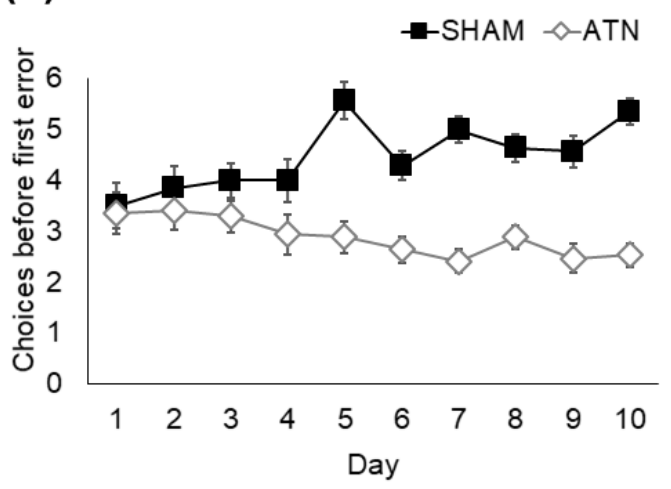

Figure 3. ATN lesions impair spatial working memory in the radial arm maze. (a) Number of errors made per day and (b) number of choices before the first error. Mean $+/$ - standard error. Sham-Lesion N=14; ATN-Lesion N=17.

(a)

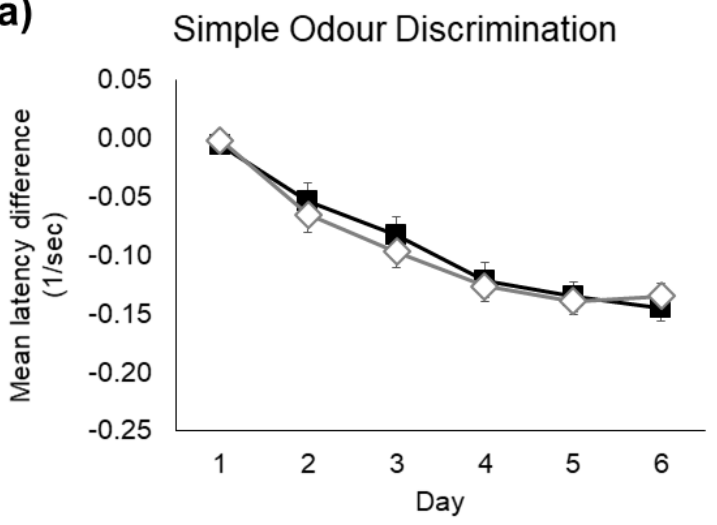

(b) Simple Object Discrimination

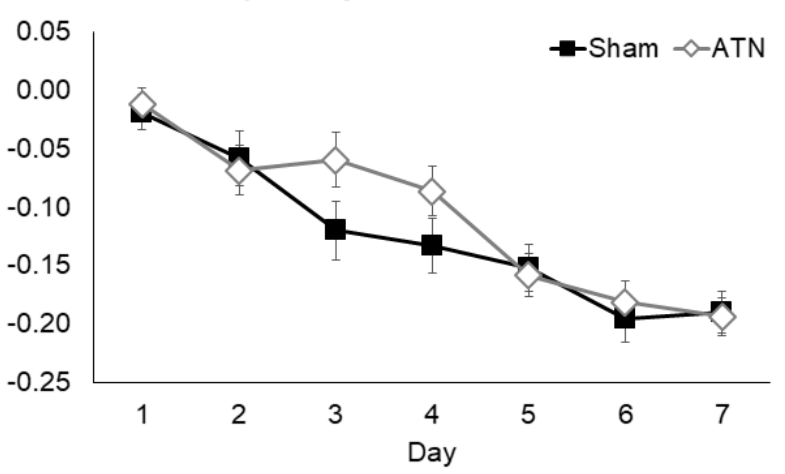

Figure 4. (a) Mean latency difference scores on the simple odour discrimination task. (b) Mean latency on the simple object discrimination task. The reciprocal latency $(1 / \mathrm{sec})$ was used because of the non-homogeneity of variance produced by raw latencies. Mean $+/-$ standard errors. Sham-Lesion N=14; ATN-Lesion N=17. 
(a)
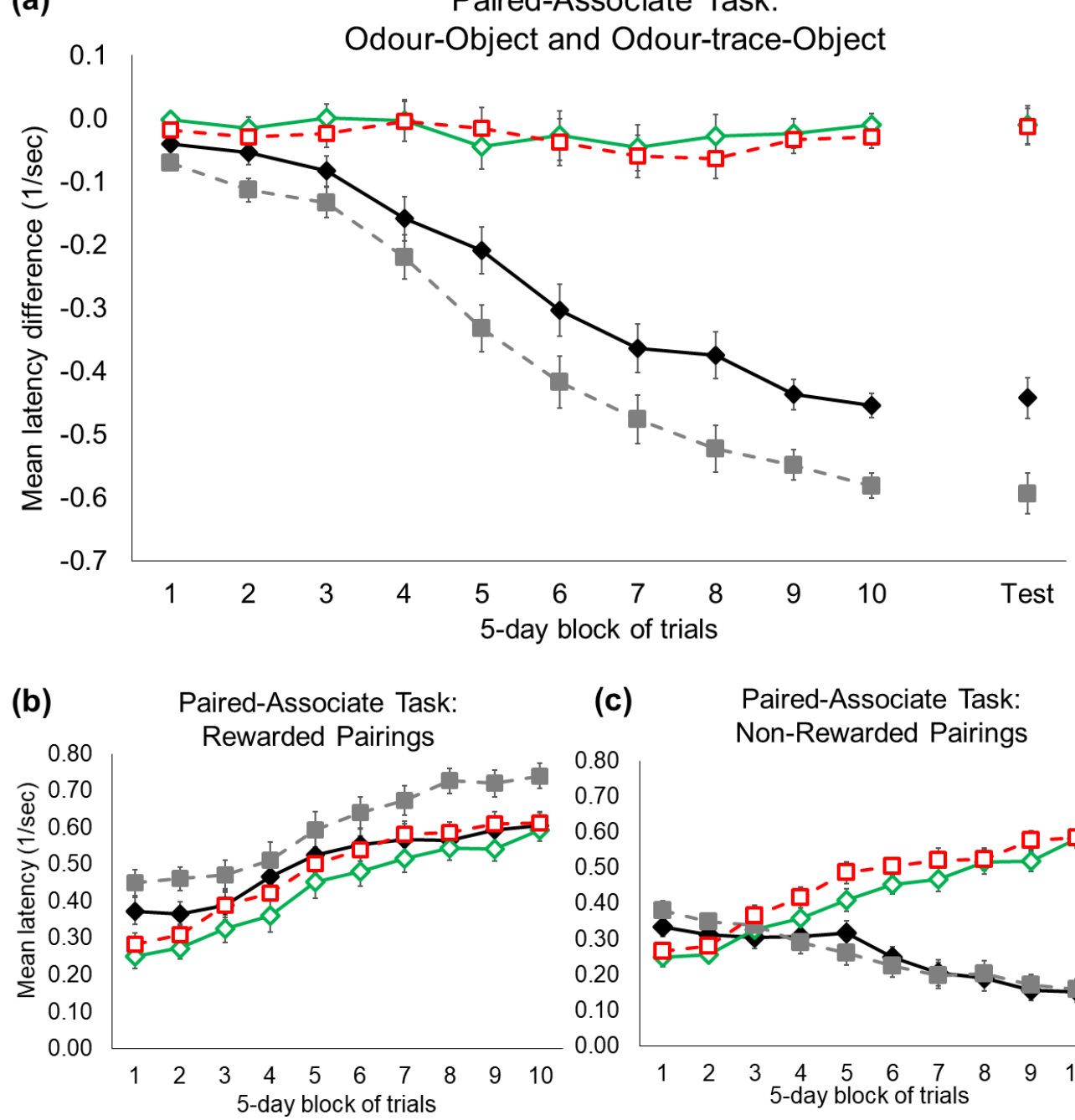

(c) Paired-Associate Task:
Non-Rewarded Pairings

Figure 5. (a) Task acquisition and recall test (5-days later) expressed as relative latency difference between rewarded and non-rewarded pairings for the odour-object (No Trace) and odour-trace-object (Trace) paired-associate learning task. Mean latency for (b) rewarded pairings and (c) non-rewarded pairings in Trace and No Trace odour-object paired-associate tasks. The reciprocal latency $(1 / \mathrm{sec})$ was used because of the non-homogeneity of variance produced by raw latencies. Mean +/- standard errors. Sham-No Trace N=7; ATN-No Trace $\mathrm{N}=8$; Sham-Trace N=7; ATN-Trace N=9. 
(a)

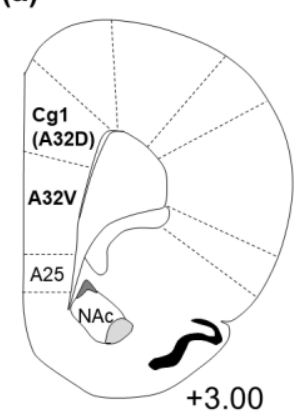

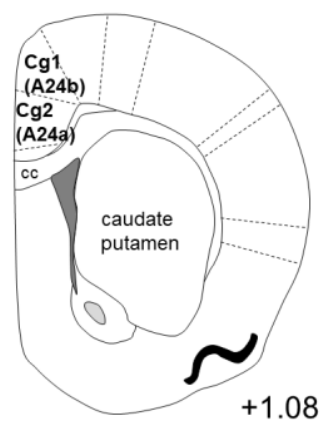

$+1.08$

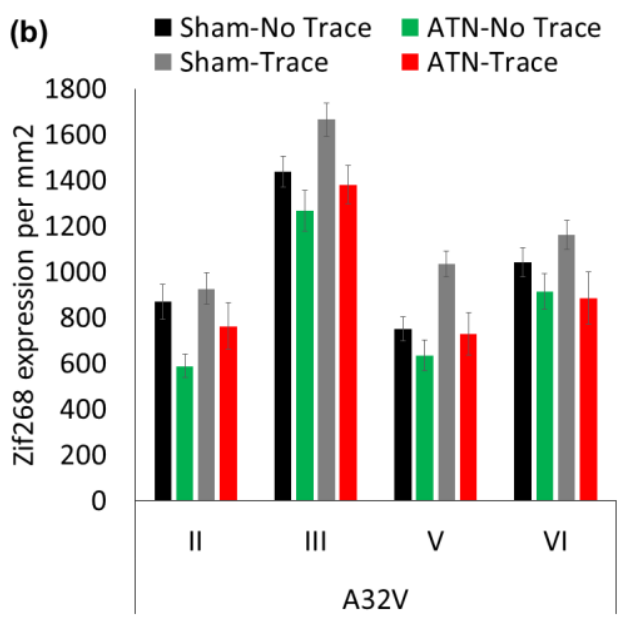

(c) 2000

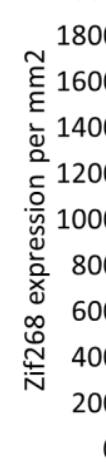

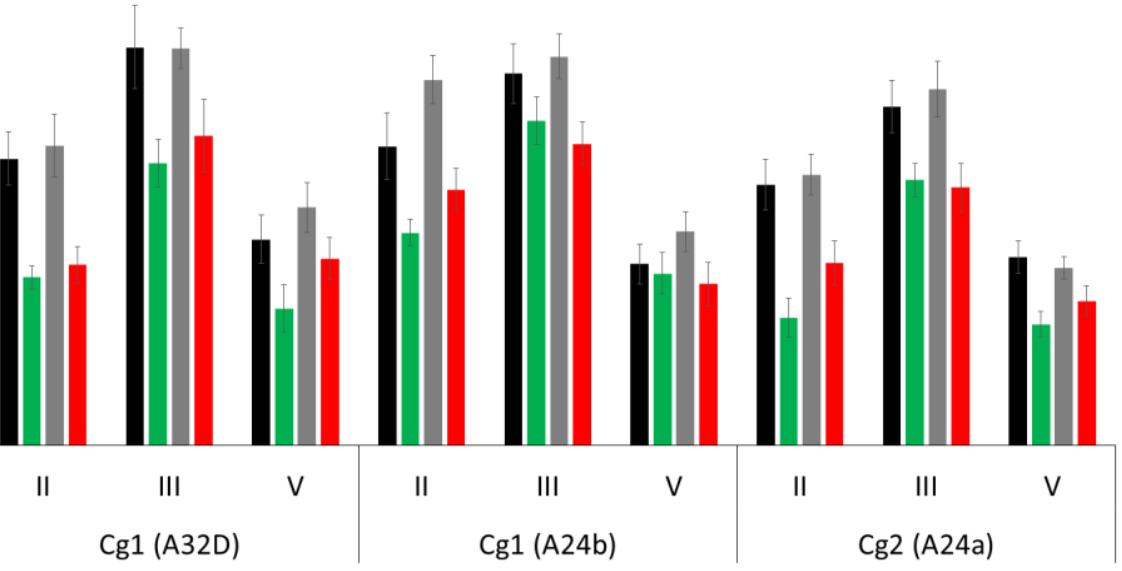

Figure 6. Zif268 expression in the prefrontal cortex regions in the four groups after the 5-day recall test. The ATN-lesion groups show decreased expression across the prefrontal regions, especially the superficial layers II and III. (a) Schematic diagram of $+3.00 \mathrm{~mm}$ and +1.08 from Bregma defining prefrontal regions. (b) Expression per mm2 in the prelimbic cortex $(\mathrm{A} 32 \mathrm{~V})$ and (c) cingulate $\mathrm{Cg} 1(\mathrm{~A} 32 \mathrm{D}$ and $\mathrm{A} 42 \mathrm{~b})$ and $\mathrm{Cg} 2$ (A24a) regions (mean \pm S.E). Sham-No Trace N=7, ATN-No Trace N=8, Sham-Trace N=7, ATN-Trace N=9. A24a = area $24 \mathrm{a}$ of the anterior cingulate cortex; $\mathrm{A} 24 \mathrm{~b}=$ area $24 \mathrm{~b}$ of the anterior cingulate cortex; $\mathrm{A} 25=$ area 25 of the prefrontal cortex (infralimbic region); $\mathrm{A} 32 \mathrm{~V}=$ area $32 \mathrm{~V}$ of the prefrontal cortex $\mathrm{A} 32 \mathrm{D}=$ area $32 \mathrm{D}$ of the anterior cingulate cortex $; \mathrm{cc}=$ corpus callosum; $\mathrm{Cg} 1=$ cingulate area $1 ; \mathrm{Cg} 2=$ cingulate area $2 ; \mathrm{NAc}=$ nucleus accumbens. 

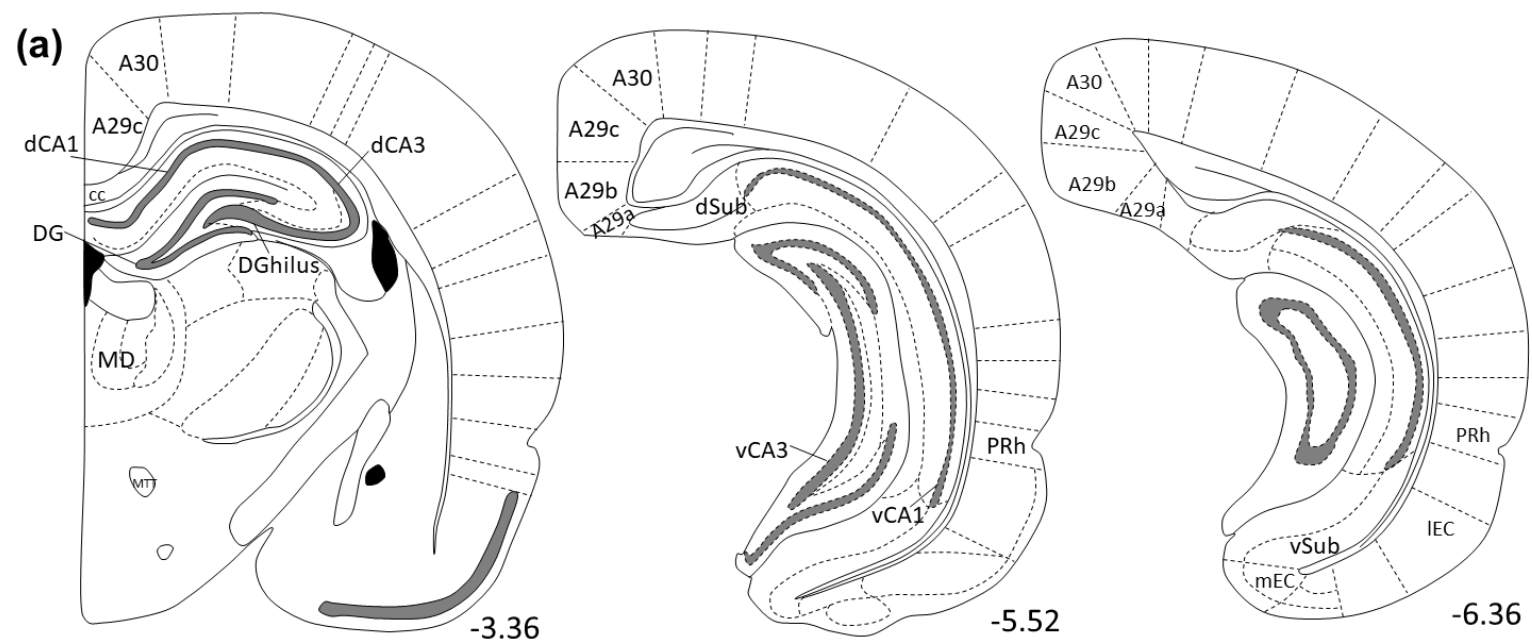

(b)

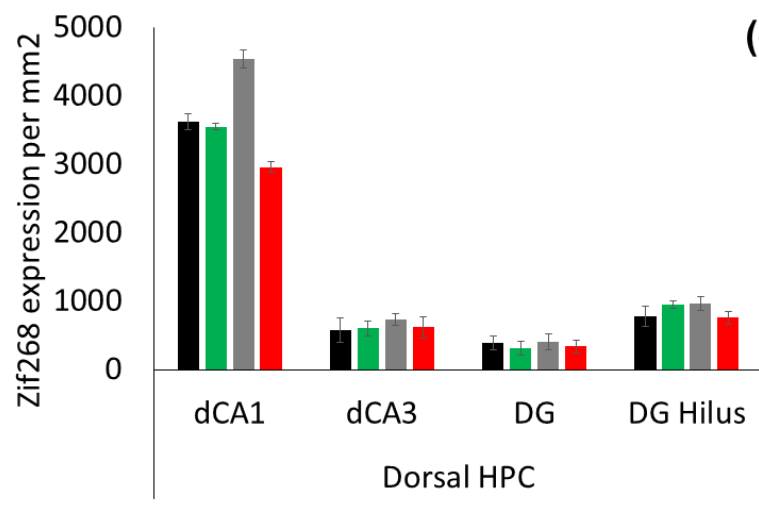

(c)

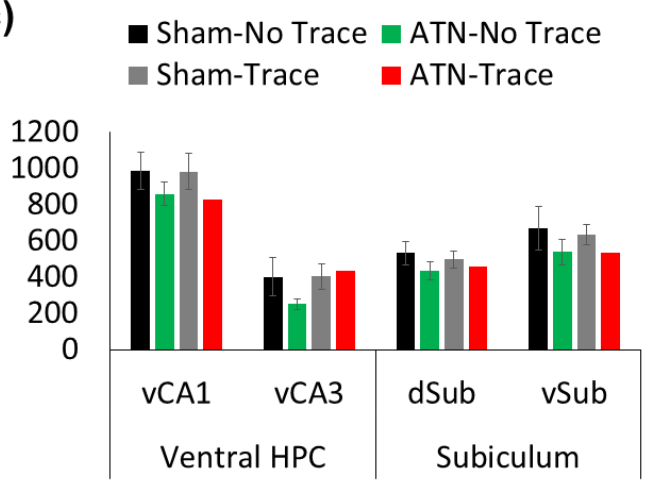

(d)
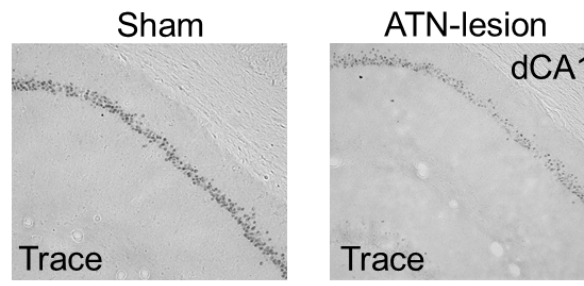

(e)
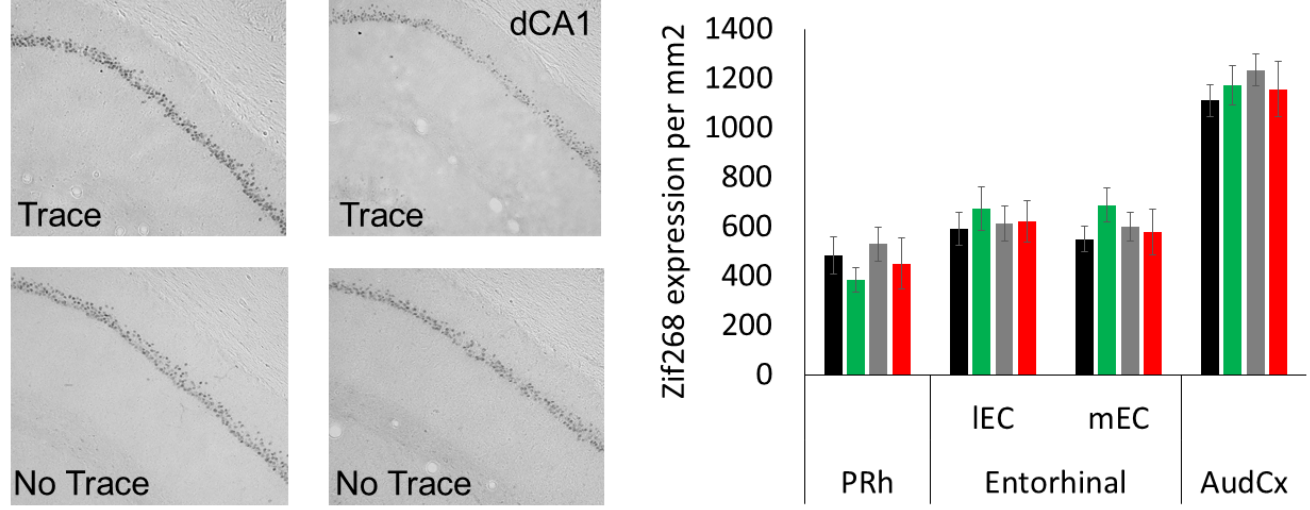

Figure 7. Zif268 in regions of the hippocampal, subicular and parahippocampal regions in the four groups after the 5-day recall test. The Sham-Trace groups showed higher Zif268 expression in the dorsal CA1. (a) Schematic example of the regions analysed (numbers represent $\mathrm{mm}$ from Bregma). (b) Expression/mm2 in the dorsal hippocampal subregions (CA1, CA3, dentate gyrus and hilus of the dentate gyrus, (c) ventral hippocampus and subiculum (CA1, CA3, dorsal and ventral subiculum) and the (e) parahippocampal regions (perirhinal, lateral and medial entorhinal and auditory cortex). Mean \pm S.E. (d) 10x 
magnification examples of Zif268 expression across Lesion and Trace conditions in the dorsal CA1 at -3.36mm from Bregma (examples from the median rat in each group). ShamNo Trace N=7, ATN-No Trace N=8, Sham-Trace N=7, ATN-Trace N=9. A29a = area 29a of the granular retrosplenial cortex; A29b = area 29b of the granular retrosplenial cortex; A29c $=\operatorname{area} 29 \mathrm{c}$ of the granular retrosplenial cortex; A30 = area 30 of the dysgranular retrosplenial cortex $; \mathrm{cc}=$ corpus callosum; $\mathrm{dCA} 1=$ dorsal cornu ammonis area $1 ; \mathrm{dCA} 3=$ dorsal $\operatorname{cornu}$ ammonis area 3; DG = dentate gyrus; DGhilus = hilus of the dentate gyrus; $\mathrm{dSub}=$ dorsal subiculum; $1 \mathrm{EC}=$ lateral entorhinal cortex $\mathrm{MD}=$ mediodorsal thalamic nuclei $; \mathrm{mEC}=$ medial entorhinal cortex $;$ MTT = mammillothalamic tract $; \mathrm{PRh}=$ perirhinal cortex $; \mathrm{vCA} 1=$ ventral cornu ammonis area 1 ; vCA3 = ventral cornu ammonis area 3; vSub = ventral subiculum. 


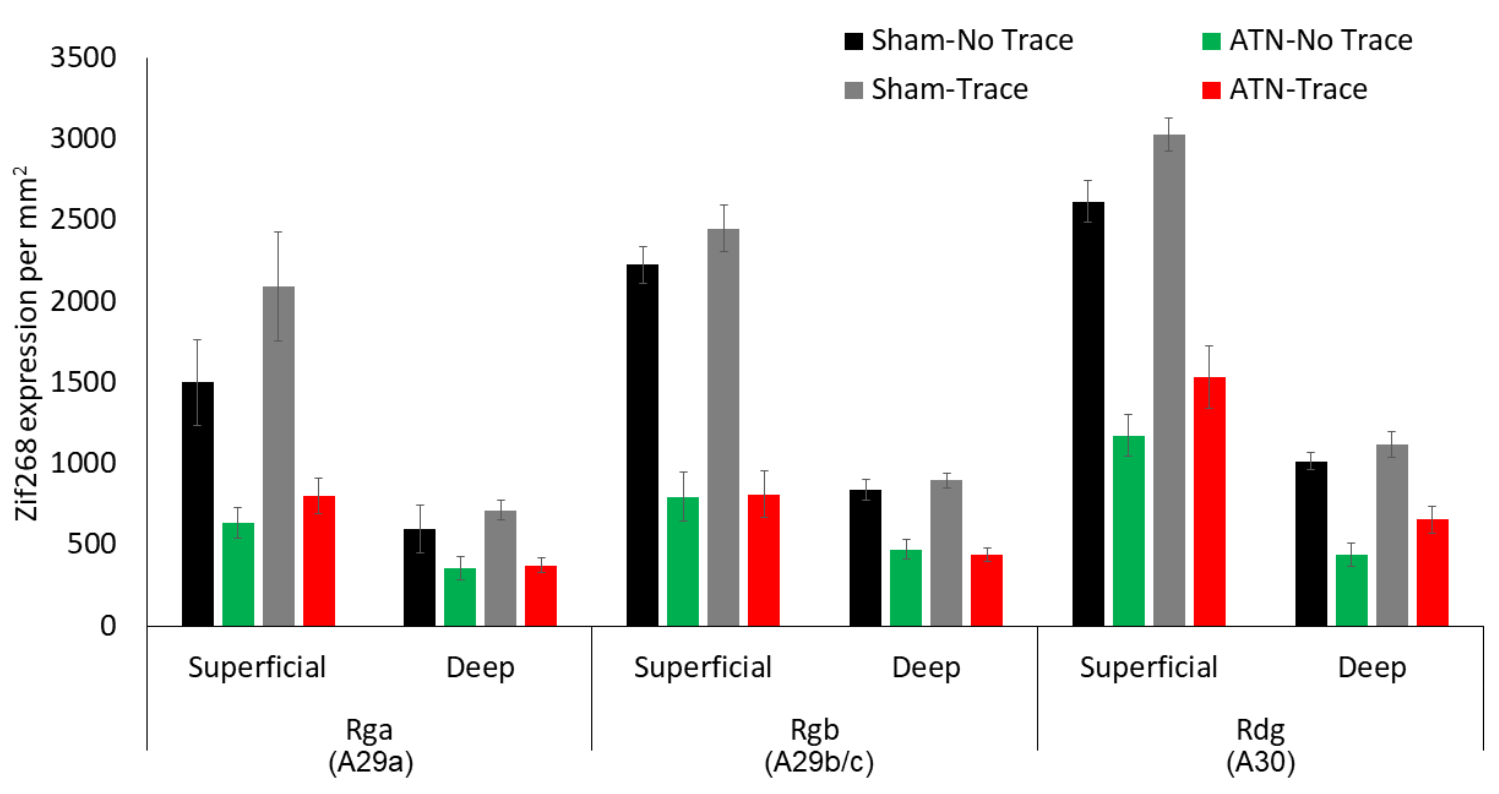

Figure 8. Zif268 expression in the retrosplenial cortex for the four groups after the 5-day recall test. ATN lesions result in a significant reduction of Zif268 across all retrosplenial regions. (a) Expression per $\mathrm{mm}^{2}$ in the granular (Rga and $\mathrm{Rgb}$ ) and dysgranular (Rdg) regions of the retrosplenial cortex (mean \pm S.E). Schematic representation of retrosplenial regions given in Figure 7. Sham-No Trace N=7, ATN-No Trace N=8, Sham-Trace N=7, ATN-Trace $\mathrm{N}=9 . \quad A 29 \mathrm{a}=$ area $29 \mathrm{a}$ of the granular retrosplenial cortex; A29b $=$ area $29 \mathrm{~b}$ of the granular retrosplenial cortex; $\mathrm{A} 29 \mathrm{c}=$ area $29 \mathrm{c}$ of the granular retrosplenial cortex; $\mathrm{A} 30=$ area 30 of the dysgranular retrosplenial cortex; Rga = dysgranular a retrosplenial cortex; Rgb $=$ dysgranular $\mathrm{b}$ retrosplenial cortex $; \mathrm{Rdg}=$ granular retrosplenial cortex . 